\title{
Anisotropic Pyrochlores and the Global Phase Diagram of the Checkerboard Antiferromagnet
}

\author{
Oleg A. Starykh, ${ }^{1}$ Akira Furusaki, ${ }^{2}$ and Leon Balents ${ }^{3}$ \\ ${ }^{1}$ Department of Physics, University of Utah, Salt Lake City, UT 84112-0830 \\ ${ }^{2}$ Condensed Matter Theory Laboratory, RIKEN, Wako, Saitama 351-0198, Japan \\ ${ }^{3}$ Department of Physics, University of California, Santa Barbara, CA 93106-4030
}

(Dated: April 28, 2005)

\begin{abstract}
We study the phase diagram of two models of spin-1/2 antiferromagnets composed of cornersharing tetrahedra, the basis of the pyrochlore structure. Primarily, we focus on the Heisenberg antiferromaget on the checkerboard lattice (also called the planar pyrochlore and crossed-chains model). This model has an anisotropic limit, when the dimensionless ratio of two exchange constants, $J_{\times} / J \ll 1$, in which it consists of one-dimensional spin chains coupled weakly together in a frustrated fashion. Using recently developed techniques combining renormalization group ideas and one-dimensional bosonization and current algebra methods, we show that in this limit the model enters a crossed dimer state with two-fold spontaneous symmetry breaking but no magnetic order. We complement this result by an approximate "quadrumer triplet boson" calculation, which qualitatively captures the physics of the "plaquette valence bond solid" state believed to obtain for $J_{\times} / J \approx 1$. Using these known points in parameter space, the instabilities pointed to by the quadrumer boson calculation, and the simple limit $J_{\times} / J \gg 1$, we construct a few candidate global phase diagrams for the model, and discuss the nature of the quantum phase transitions contained therein. Finally, we apply our quasi-one-dimensional techniques to an anisotropic limit of the threedimensional pyrochlore antiferromagnet, an approximate model for magnetism in $\mathrm{GeCu}_{2} \mathrm{O}_{4}$. A crossed dimer state is predicted here as well.
\end{abstract}

\section{INTRODUCTION}

We consider one of the most frustrated twodimensional (2D) antiferromagnets: the checkerboard antiferromagnet, also known as the planar pyrochlore and the crossed-chains model (CCM). As the name suggests, this model is motivated by the three-dimensional (3D) pyrochlore materials. The $2 \mathrm{D}$ model is obtained by a projection of $3 \mathrm{D}$ corner-sharing lattice of tetrahedra on a 2D plane. This projection maps a four-spin tetrahedron onto a four-spin square with additional links (antiferromagnetic exchanges) along the diagonals. The structure obtained in this way, depicted in Fig. 1 preserves the corner-sharing arrangement of crossed squares, typical of the original 3D pyrochlore lattice, but destroys the symmetry between bonds of the tetrahedron: in two dimensions, the horizontal and vertical bonds are not equivalent to diagonal ones. This lowering of symmetry suggests consideration of extended 2D models with the checkerboard structure where exchange interactions on horizontal/vertical and diagonal bonds take on different values. Among these, the quasi-one-dimensional limit, in which exchange along horizontal and vertical directions $J$ is much stronger than that along diagonal directions $J_{\times}$, is of special interest because it involves competition between strong quantum fluctuations, typical for one-dimensional (1D) spin chains, and equally strong geometric frustration encoded in the structure of the crossed-chains lattice.

The resulting checkerboard antiferromagnet has been analyzed by a variety of techniques along several complimentary "directions" in the parameter space: semiclassical analysis in the limit of large spin $S \gg$

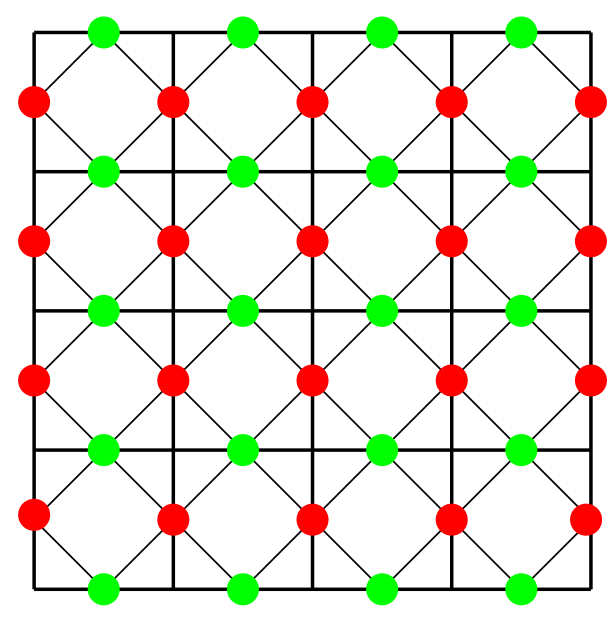

FIG. 1: (Color online) Heisenberg antiferromagnet on the checkerboard lattice, viewed as coupled spin chains. Horizontal (vertical) spin chains run along the $x(y)$ axis. Spins belonging to the horizontal (vertical) chains are shown as green (red) filled circles. The intra-chain exchange (thick lines) is $J$, and the inter-chain exchange (diagonal thin lines) is $J_{\times}$.

$1, \stackrel{1,2.3}{,}$ large- $N$ expansion,$\stackrel{4.5 .6}{,}$ easy-axis generalization (of the $3 \mathrm{D}$ model) $\frac{7}{\underline{7}}$ and a quasi-1D $\left(J_{\times} / J \ll 1\right)$ approach $\stackrel{8}{*}$ In parallel with analytic approaches, the model was investigated numerically via exact diagonalization studies.10.11 and cluster-based strong-coupling expansion techniques ${ }^{12,13,14}$ The present paper complements these approaches by combining a controlled analysis of the quasi-1D limit with general arguments to pin down limits of the phase diagram and postulate a likely global phase structure of the model. 
We begin by expounding the more general context of the problem. One of the central theoretical motivations behind the study of frustrated quantum magnets is the hope that, when magnetic ordering is suppressed by frustration, more novel types of order or even criticality may emerge. Phenomenological approaches suggest possible interesting quantum phases exhibiting "valence bond solid" (VBS) order, in which spins pair into singlets that are spontaneously localized on specific bonds, breaking lattice symmetries. More exotically, such approaches suggest the possibility of phases with "topological order", in which spins fluctuate quantum mechanically in a liquid-like state with however subtle topological properties and often excitations with anomalous (e.g., fractional) quantum numbers. More recent predictions from such theories also include "deconfined" quantum critical points and phases in which several types of quasi-longrange (power-law) orders coexist unconnected by microscopic symmetries.

Unfortunately, these types of phenomenological methods do not give precise guidance as to the specific models in which such quantum orders appear, and attempts to find them in realistic microscopic Hamiltonians have met with at best limited success. The one specific context in which examples of all the above phenomena are, however, known to occur is in one-dimensional spin chains. Moreover, the theoretical and microscopic understanding of such spin models is vastly more complete than in two or three dimensions. A natural hunting ground for the exotic phenomenology described above would hence seem to lie in spin models consisting of chains weakly coupled into two or three dimensional arrays. A recently gained understanding of the crucial role of nominally irrelevant operators and fluctuation-generated interactions in describ-

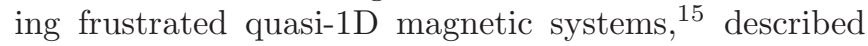
below, brings the hunt to (some degree of) fruition.

In this paper, as in a previous work $\stackrel{15}{15}$ we follow this approach, taking as the weakly coupled units in question $S=1 / 2$ Heisenberg nearest-neighbor antiferromagnetic chains (other further-neighbor interactions along each chain may be included, provided they are not overly strong). A cause for hope is that such a 1D chain is well known to exhibit a critical ground state with power-law correlations of various types. One prominent type of correlation in such a chain is antiferromagnetic, specifically:

$$
\left\langle\vec{S}(n) \cdot \vec{S}\left(n^{\prime}\right)\right\rangle \sim \frac{(-1)^{n-n^{\prime}}}{\left|n-n^{\prime}\right|}+\cdots,
$$

where $n$ is the coordinate along the chain, and the brackets indicate a ground state expectation value. The omitted $\cdots$ terms decay much faster $\left(\sim 1 /\left|n-n^{\prime}\right|^{2}\right.$ or faster $)$ than the dominant slowly-decaying antiferromagnetic one shown here (we have also for simplicity neglected an unimportant multiplicative logarithmic correction to this term). The dominance of antiferromagnetic correlations in the two-spin correlation function often leads to the misconception that a good picture of the ground state of the $1 \mathrm{~d}$ Heisenberg chain is that of fluctuating local antiferromagnetic order, i.e., a magnet in which spins are locally Néel ordered but the quantization axis fluctuates in space and time. Such a picture is in fact incomplete. This becomes clear upon considering the fluctuation of the local bond energy or dimerization,

$$
B(n)=\vec{S}(n) \cdot \vec{S}(n+1)-\langle\vec{S}(n) \cdot \vec{S}(n+1)\rangle .
$$

One finds that its (staggered) correlations,

$$
\left\langle B(n) B\left(n^{\prime}\right)\right\rangle \sim \frac{(-1)^{n-n^{\prime}}}{\left|n-n^{\prime}\right|}+\cdots,
$$

have precisely the same slow power-law decay (again, up to a multiplicative logarithmic correction) as the antiferromagnetic ones in Eq. (11) above! Further examination of other correlators reveals no additional power-law correlations with competitive slow decay. Thus the 1D Heisenberg chain should be thought of as consisting of locally fluctuating antiferromagnetic and valence bond solid order of comparable strength.

With this understanding, it is natural to expect that weakly-coupled arrays of such chains might be pushed by the inter-chain coupling into magnetically ordered, dimer ordered, or perhaps critical states, if this coupling favors the intrinsic antiferromagnetic or VBS ordering tendency, or fosters their balanced competition, respectively. While we believe this reasoning to be essentially correct, for many years, the richness of such possible behaviors went unrealized in the literature. This is because if the spin chains are linked by magnetic two-spin Heisenberg interactions, these couple primarily to the antiferromagnetic fluctuations within the chains, and not to the VBS ones. Hence, for such a case, the problem of Heisenberg spin chains coupled by weak inter-chain interactions is rather well understood. With non-frustrated transverse (with respect to the chain direction) couplings, both renormalization group 16 and self-consistent mean-field analysis 17 predict an instability towards classical long-range ordered phase characterized by a non-zero expectation value of the spin $\left\langle\vec{S}_{r}\right\rangle \neq 0$. This instability follows from the correlations in Eq. (11), which, loosely speaking, make the spin chain highly susceptible to magnetic ordering.

More recently, it was recognized that the situation becomes more interesting and less clear-cut when the interchain interaction is strongly frustrated, as is the case for the crossed-chains model we investigate here. The effect of frustration is to reduce and, ultimately, nullify the effective inter-chain magnetic field experienced by spins of the chain due to transverse inter-chain exchange interactions (due to cancellations between contributions from spins whose local fluctuating orientations, according to Eq. (10), are antiparallel). With no effective external field present, the classical ordering instability is naturally absent, resulting in (almost) decoupled behavior of distinct spin chains. In formal calculations embodying this physical picture, the weak residual 
inter-chain interaction which does not cancel with predominantly antiferromagnetic correlations appears to be described by the scalar product of conserved spin currents from the chains involved. This observation led to the proposal that, as a result, the system of such coupled chains forms a liquid-like ground state with fractionalized spin excitations (spinons). The systems considered included a frustrated spin ladder $\stackrel{18.19}{=}$ its $2 \mathrm{D}$ extension, i.e., the spatially-anisotropic frustrated square lattice antiferromagnet,, 20 and the crossed-chains model ${ }^{8}$

As shown in Ref. 15, in the former two cases these conclusions are in fact incorrect, due to the neglect of the VBS correlations, Eq. (3), equally as inherent as the antiferromagnetic ones to the Heisenberg chain. Although the microscopic magnetic exchange between spins on different chains does not directly couple to VBS fluctuations, such a dimer coupling between (certain pairs of) chains is inevitably generated by the weak residual magnetic interactions remaining after the dominant antiferromagnetic cancellation. A careful analysis of the types of such dimer couplings allowed by symmetry and the detailed mechanism of their generation are crucial in determining the fate of the spin system and the strength of any ordering tendency.

Technically, this analysis can be accomplished in a controlled fashion using powerful field-theoretical methods borrowed from 1D physics. The point, made in Ref. 15, is that no fine-tuning of the two-spin inter-chain exchange interaction can make the low-energy field theory exactly of current-current type. Some higher-order derivative terms (typically involving spatial derivatives of the staggered magnetization field) are bound to be present (as getting rid of all of them to all orders would require tuning infinite number of inter-chain couplings to zero). Such derivative terms are commonly neglected on the grounds of their irrelevance with respect to the Luttinger liquid fixed point of the independent spin chain. However, the quasi-1D problem is not the same as the purely 1D one. Instead of disregarding irrelevant highderivatives terms from the outset, one has to consider if they, in combination with the leading current-current term, can produce quantum corrections to the relevant inter-chain couplings. This indeed occurs both in the models of Ref. 15, and, as we will see, in the crossedchains model studied here.

In the present paper we extend the analysis of Ref. 15 to the CCM and show that previous claim of the sliding Luttinger liquid ground state ${ }^{\underline{\underline{\underline{ }}}}$ is not correct. Instead, similarly to the spatially-anisotropic square lattice model discussed above, the ground state is of spontaneously dimerized type, albeit with staggered ordering of dimers on parallel chains. The resulting configuration, shown in Fig. 2] can be described as a crossed-dimer one.

The paper is organized as follows. Section $\coprod$ describes the Hamiltonian of the CCM model, its lattice symmetries, and the passage to the field-theoretical description of the low-energy degrees of freedom and the operator product algebra they form. Section III describes pertur-

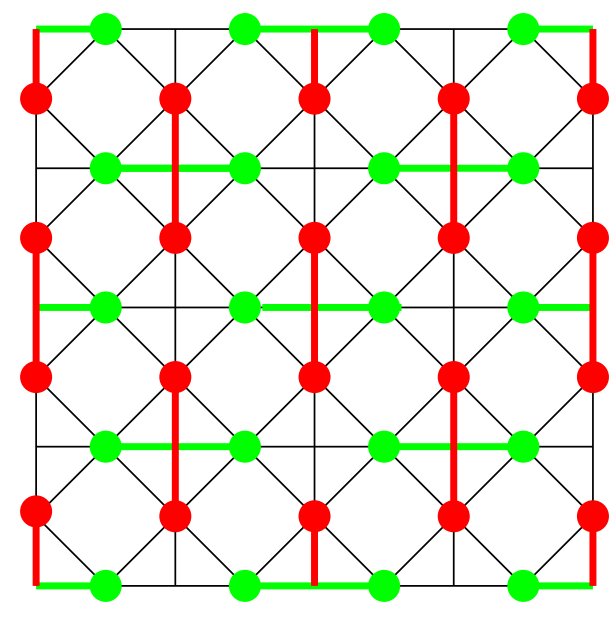

FIG. 2: (Color online) Crossed-dimer dimerization pattern. "Strong" bonds (ones where $\epsilon>0$ ) on horizontal (vertical) chains are shown in green (red). As before, spins on horizontal (vertical) chains are denoted by green (red) circles.

bative analysis of the model in the one-dimensional limit of weakly coupled chains, $J_{\times} / J \ll 1$. It contains key technical details of our work and explains the mechanism by which the crossed-dimer phase is stabilized. The limit of the fully two-dimensional model $\left(J_{\times} \approx J\right)$, the planar pyrochlore antiferromagnet, is analyzed within the plaquette-operator mean-field approximation in Sec. IV This is followed by Sec. $\mathbb{\nabla}$ which summarizes the preceding material in terms of two possible scenarios for the global zero-temperature phase diagram of the checkerboard antiferromagnet. There we present phenomenological symmetry-based analyses of the quantum phase transitions between various phases of the model (and also point out an interesting connection with the recent deconfined quantum critical point idea). Section VI describes a three-dimensional extension of our model, the quasi-one-dimensional pyrochlore antiferromagnet, and its possible relevance to the experiments on $\mathrm{GeCu}_{2} \mathrm{O}_{4}$ and $\mathrm{ZnV}_{2} \mathrm{O}_{4}$. Our main points are briefly summarized in Sec. VII Two Appendices contain important technical details of the fermionic formulation of the low-energy sector of the $S=1 / 2$ isotropic Heisenberg chain.

\section{FROM LATTICE TO CONTINUUM FIELD THEORY}

\section{A. Lattice model and symmetries}

The Hamiltonian of the system $H$ describes a collection of horizontal $\left(H_{h}\right)$ and vertical $\left(H_{v}\right)$ Heisenberg chains interacting with each other via the inter-chain interaction $V$ :

$$
H=H_{0}+V=H_{h}+H_{v}+V .
$$


Spins $(S=1 / 2)$ are located at the sites of the checkerboard (crossed-chains) lattice shown in Fig. 1 The crossings of the lattice have integer coordinates $(n, m)$, so the sites of horizontal chains have half-integer $x$-coordinates $n+\frac{1}{2}$ and integer $y$-coordinate $m$, while sites of the vertical chains are described by $\left(n, m+\frac{1}{2}\right)$ pairs. With this convention the Hamiltonian of horizontal chains reads

$$
H_{h}=J \sum_{n, m} \vec{S}_{h}(n-1 / 2, m) \cdot \vec{S}_{h}(n+1 / 2, m) .
$$

Similarly, $H_{v}$ is given by

$$
H_{v}=J \sum_{n, m} \vec{S}_{v}(n, m-1 / 2) \cdot \vec{S}_{v}(n, m+1 / 2) .
$$

With local uniform magnetization defined by

$$
\begin{aligned}
& \vec{s}_{h}(n, m)=\vec{S}_{h}(n-1 / 2, m)+\vec{S}_{h}(n+1 / 2, m), \\
& \vec{s}_{v}(n, m)=\vec{S}_{v}(n, m-1 / 2)+\vec{S}_{v}(n, m+1 / 2),
\end{aligned}
$$

the inter-chain interaction reads

$$
V=J_{\times} \sum_{n, m} \vec{s}_{h}(n, m) \cdot \vec{s}_{v}(n, m)
$$

and is characterized by the inter-chain exchange $J_{\times}>0$ which is much smaller than the in-chain antiferromagnetic exchange $J>0$. We note that $J_{\times}$is the nearestneighbor exchange on the checkerboard lattice while $J$ is the next-nearest-neighbor exchange interaction.

The space group symmetries of $H$, Eq. (44), can now be summarized. The translational subgroup is generated by unit translation along the horizontal chains $T_{h}$ and that along the vertical chains $T_{v}$. The remainder is generated by $\pi / 2$ rotations about a crossing, and reflections about e.g., a vertical line through either a site or midpoint of a bond of a horizontal chain. We denote these two operations "site parity" $P_{s h}$ and "link parity" $P_{L h}$, respectively. As these are microscopic lattice symmetries, they will be preserved by any renormalization group transformation. Observe that $P_{L}$ is a product of two other operations: $P_{L h}=P_{s h} \circ T_{h}$.

\section{B. Continuum field theory and scaling operators}

The limit $J_{\times} \ll J$ allows us to approach the problem from one-dimensional perspective: we treat $V$ as a perturbation and ask whether it can destabilize the critical ground state of the independent (decoupled) spin chains. The smallness of the $J_{\times} / J$ ratio allows us to take the continuum limit along every chain involved. As mentioned in the Introduction, a single Heisenberg chain is described in the continuum limit (i.e., at low energies) by a universal critical theory, with a variety of power-law correlations. Formally, this is most compactly described as the Wess-Zumino-Witten (WZW) $S U(2)_{1}$ theory ${ }^{21}$, with the action (in $1+1$ dimensions) $)^{22,23}$

$$
\begin{aligned}
S_{W Z W}= & \frac{1}{8 \pi} \int d^{2} x \operatorname{Tr} \partial_{\mu} g^{\dagger} \partial_{\mu} g \\
& -\frac{i}{12 \pi} \int d^{3} x \epsilon_{\mu \nu \lambda} \operatorname{Tr} g^{\dagger} \partial_{\mu} g g^{\dagger} \partial_{\nu} g g^{\dagger} \partial_{\lambda} g .
\end{aligned}
$$

Here $g$ is an $S U(2)$ matrix. The coordinate $x_{0}=v \tau(v$ is the spin velocity and $\tau$ is imaginary time) and $x_{1}=x$, the coordinate along the chain, and $d^{3} x$ is defined by extending this $2 \mathrm{D}$ space into a three-dimensional hemisphere $x_{2}<0$, the boundary of which is the (compactified) physical 2D plane $\left(x_{0}, x_{1}\right)$, and analytically continuing $g\left(x_{0}, x_{1}\right)$ into this hemisphere such that $g\left(x_{0}, x_{1}, x_{2} \rightarrow\right.$ $-\infty) \rightarrow 1$ and $g\left(x_{0}, x_{1}, x_{2}=0\right)=g\left(x_{0}, x_{1}\right)$. This formal action is not of very much direct practical use, but serves to illustrate the underlying degrees of freedom of the critical theory. All operators in the WZW $S U(2)_{1}$ theory can be constructed from $g$. Corresponding to the two dominant power-law correlations in Eqs. (1) and (3), there are two scaling operators 22.23

$$
\begin{aligned}
\vec{N} & \sim-i \operatorname{Tr} g \vec{\sigma}, \\
\epsilon & \sim \operatorname{Tr} g .
\end{aligned}
$$

Here $\vec{\sigma}$ is the vector of Pauli matrices, and the $\sim$ indicates that the proportionality between these fields and the physical staggered magnetization/dimerization involves a cut-off dependent factor. The operator $\vec{N}$ represents the local staggered magnetization, while $\epsilon$ represents the local staggered dimerization (it is the continuum version of the bond operator in (2)). There are also subdominant power-law correlations arising from fluctuations of the chiral $S U(2)$ currents,

$$
\begin{aligned}
& \vec{J}_{R}=\frac{1}{4 \pi} \operatorname{Tr} g^{\dagger} \bar{\partial} g \vec{\sigma}, \\
& \vec{J}_{L}=\frac{1}{4 \pi} \operatorname{Tr} g \partial g^{\dagger} \vec{\sigma},
\end{aligned}
$$

with $\partial=\left(\partial_{0}-i \partial_{1}\right) / 2$, and $\bar{\partial}=\left(\partial_{0}+i \partial_{1}\right) / 2$. Physically, the operator $\vec{J}=\vec{J}_{R}+\vec{J}_{L}$ represents the local uniform magnetization, while $v\left(\vec{J}_{R}-\vec{J}_{L}\right)$ represents the local magnetization (spin transport) current.

All the low-energy power-law correlations of the weakly coupled Heisenberg chains can be exposed by decomposing lattice operators into a set of the above continuum operators (and generally their derivatives, see below) for each chain. This, for example, leads to the following decomposition of the spin at a site $n-1 / 2$ along the horizontal chain number $m$ :

$$
\vec{S}_{h}(n-1 / 2, m)=a\left[\vec{J}_{h, m}(x)+(-1)^{n} \vec{N}_{h, m}(x)\right] .
$$

Here $x=n a$ ( $a$ is the lattice spacing) and $\vec{J}$ (respectively, $\vec{N}$ ) represents uniform (respectively, staggered) part of the spin density. Similarly, for the vertical spin chains we have

$$
\vec{S}_{v}(n, m-1 / 2)=a\left[\vec{J}_{v, n}(y)+(-1)^{m} \vec{N}_{v, n}(y)\right],
$$


where $y=m a$. Notice that the continuum limit is taken only for the coordinate along the chain; the perpendicular one becomes an index $m$ (respectively, $n$ for vertical chains). The uniform spin magnetization $\vec{J}$ is the sum of the right $\left(\vec{J}_{R}\right)$ and left $\left(\vec{J}_{L}\right)$ moving components, $\vec{J}=\vec{J}_{R}+\vec{J}_{L}$, and represents the conserved spin density (it is often referred to in the literature as the spin "current", the term originating from the relativistic concept of space-time current, whose time component is the conserved density). Note that the staggered dimerization $\epsilon$ does not appear in Eq. (14); in fact, it cannot appear in the decomposition of any single spin operator since it is not a vector under $S U(2)$. As discussed in the Introduction, for this reason dimer order does not appear likely in weakly coupled Heisenberg chains with unfrustrated inter-chain couplings.

The action of the microscopic space group symmetries (described above) upon the continuum scaling operators will be crucial in the following. These are rather clear on physical grounds 24 :

Translation:

$$
T: \vec{J} \rightarrow \vec{J}, \quad \vec{N} \rightarrow-\vec{N}, \quad \epsilon \rightarrow-\epsilon
$$

Site parity:

$$
P_{s}: \vec{J} \rightarrow \vec{J}, \quad \vec{N} \rightarrow \vec{N}, \quad \epsilon \rightarrow-\epsilon .
$$

Link parity: Using $P_{L}=P_{s} \circ T$ we find

$$
P_{L}: \vec{J} \rightarrow \vec{J}, \quad \vec{N} \rightarrow-\vec{N}, \quad \epsilon \rightarrow \epsilon .
$$

We will see at the end of this section that this symmetry is responsible for the absence of $\vec{N}_{v} \cdot \vec{N}_{h}$ terms in the Hamiltonian of the problem.

Because of somewhat non-intuitive point-splitting identities, the WZW model can be written in Hamiltonian form (known as the Sugawara form) in terms of the spin currents. For a single chain, one has

$$
H_{W Z W}=\frac{2 \pi v}{3} \int d x\left[\vec{J}_{R}(x) \cdot \vec{J}_{R}(x)+\vec{J}_{L}(x) \cdot \vec{J}_{L}(x)\right] .
$$

Applied to the set of horizontal chains (labelled by $m$ ), the lattice Hamiltonian $H_{h}$, Eq. (5), transforms into

$$
\begin{aligned}
H_{h}=\frac{2 \pi v}{3} \sum_{m} \int d x & {\left[\vec{J}_{h, m, R}(x) \cdot \vec{J}_{h, m, R}(x)\right.} \\
& +\vec{J}_{h, m, L}(x) \cdot \vec{J}_{h, m, L}(x) \\
& \left.+g_{b s} \vec{J}_{h, m, R}(x) \cdot \vec{J}_{h, m, L}(x)\right] .
\end{aligned}
$$

Here $v=\frac{\pi}{2} J a$ is the spin velocity. Note again that $J_{h, m, R / L}^{a}(x)$ depends on position $x=n a$ along the chain direction whereas its $y=m a$ coordinate dependence only shows up via the (horizontal) chain index $m$. We have actually included in Eq. (20) a correction (proportional to $\left.g_{b s}\right)$ to the WZW model, which is present in the Heisenberg chain but is marginally irrelevant in the situation under consideration. For this reason, it may be safely neglected in what follows. Similarly

$$
\begin{aligned}
H_{v}=\frac{2 \pi v}{3} \sum_{n} \int d y & {\left[\vec{J}_{v, n, R}(y) \cdot \vec{J}_{v, n, R}(y)\right.} \\
& +\vec{J}_{v, n, L}(y) \cdot \vec{J}_{v, n, L}(y) \\
& \left.+g_{b s} \vec{J}_{v, n, R}(y) \cdot \vec{J}_{v, n, L}(y)\right] .
\end{aligned}
$$

\section{Decomposition of the full lattice model}

Now we are ready to express the inter-chain perturbation Eq. (8) in terms of low-energy modes $\vec{J}$ and $\vec{N}$. We begin by analyzing the sum of two neighboring spins on the same (say, horizontal) chain,

$$
\begin{aligned}
\vec{s}_{h}(n, m) & =\vec{S}_{h}(n-1 / 2, m)+\vec{S}_{h}(n+1 / 2, m) \\
& =a\left[2 \vec{J}_{h, m}(x)-(-1)^{n} a \partial_{x} \vec{N}_{h, m}(x)\right] .
\end{aligned}
$$

For the reasons to be explained in detail below, we have retained the next-to-leading irrelevant contribution $\left(\partial_{x} \vec{N}\right)$ in this expression. Similar decomposition is done for the sum of two spins on the crossing vertical chain. The interchain interaction $V$ thus reads

$$
\begin{aligned}
V=\sum_{n, m}\{ & g_{j j} \vec{J}_{h, m}(x) \cdot \vec{J}_{v, n}(y) \\
& \quad-g_{n j}\left[(-1)^{n} \partial_{x} \vec{N}_{h, m}(x) \cdot \vec{J}_{v, n}(y)\right. \\
& \left.\quad+(-1)^{m} \vec{J}_{h, m}(x) \cdot \partial_{y} \vec{N}_{v, n}(y)\right] \\
& \left.+g_{n n}(-1)^{n+m} \partial_{x} \vec{N}_{h, m}(x) \cdot \partial_{y} \vec{N}_{v, n}(y)\right\}
\end{aligned}
$$

where, as before, $x=n a, y=m a$ and the following couplings are introduced to shorten notations:

$$
g_{j j}=4 J_{\times} a^{2}, \quad g_{n j}=2 J_{\times} a^{3}, \quad g_{n n}=J_{\times} a^{4} .
$$

It is important to observe that Eq. (23) does not contain $\vec{N}_{h} \cdot \vec{N}_{v}$ type of terms, which are forbidden by the symmetry of the checkerboard lattice. For example, reflection with respect to the vertical chain changes sign of $\vec{N}_{h},\left(P_{L}: \vec{N}_{h} \rightarrow-\vec{N}_{h}\right)$, while leaving $\vec{N}_{v}$ invariant, see Eq. (18). This reflects strong frustration of the model under study, as discussed in the Introduction. Observe also that any pair of horizontal and vertical chains cross only once, which makes Eq. (23) local in space. This requires us to think carefully about the short-distance regularization of the low-energy theory defined by Eq. (20), Eq. (21), and Eq. (23) - the corresponding analysis is described in the next Section.

\section{Operator product expansion}

Various perturbations to the WZW model Eq. (19) [such as the intra-chain backscattering $g_{b s}$ in Eq. (20) 
and Eq. [21), and the inter-chain $V$, Eq. [23)] are most conveniently analyzed with the help of operator product expansions (OPE). These are operator identities that are derived by applying Wick's theorem to a correlation function of a pair of operators at nearby points, say, $(x, \tau)$ and $(0,0)$ - several of the examples below are worked out in Appendix B see also Appendix A of Ref. 25 for more examples. The OPE below are valid for operators from the same chain, and, to lighten expressions, we suppress chain indices here.

The spin currents $\vec{J}_{R / L}$ obey the following chiral OPEs, which are frequently used in the literature ${ }^{23}$ [these, for example, are used to derive the renormalization-group flow of $g_{b s}$ term in Eqs. (20) and (21)]:

$$
\begin{aligned}
J_{R}^{a}(x, \tau) J_{R}^{b}(0)= & \frac{\delta^{a b}}{8 \pi^{2} v^{2}\left(\tau-i x / v+\alpha \sigma_{\tau}\right)^{2}} \\
& +\frac{i \epsilon^{a b c} J_{R}^{c}(0)}{2 \pi v\left(\tau-i x / v+\alpha \sigma_{\tau}\right)}
\end{aligned}
$$

and

$$
\begin{aligned}
J_{L}^{a}(x, \tau) J_{L}^{b}(0)= & \frac{\delta^{a b}}{8 \pi^{2} v^{2}\left(\tau+i x / v+\alpha \sigma_{\tau}\right)^{2}} \\
& +\frac{i \epsilon^{a b c} J_{L}^{c}(0)}{2 \pi v\left(\tau+i x / v+\alpha \sigma_{\tau}\right)},
\end{aligned}
$$

where, as explained in Appendix A2 $\alpha=a / v$ is the short-time cutoff of the theory and $\sigma_{\tau}=\operatorname{sign}(\tau)$.

Being a conserved current, $J^{a}$ is also a generator of rotations. Thus the OPE of $J^{a}$ and $N^{a}$ should be nontrivial. In fact, this one is the most important OPE for the subsequent analysis (see Appendix $\mathbb{B}$ for the derivation),

$$
\begin{aligned}
& J_{R}^{a}(x, \tau) N^{b}(0)=\frac{i \epsilon^{a b c} N^{c}(0)-i \delta^{a b} \epsilon(0)}{4 \pi v\left(\tau-i x / v+\alpha \sigma_{\tau}\right)}, \\
& J_{L}^{a}(x, \tau) N^{b}(0)=\frac{i \epsilon^{a b c} N^{c}(0)+i \delta^{a b} \epsilon(0)}{4 \pi v\left(\tau+i x / v+\alpha \sigma_{\tau}\right)} .
\end{aligned}
$$

Finally, fusing spin current with dimerization $\epsilon$ gives back the staggered magnetization

$$
\begin{aligned}
& J_{R}^{a}(x, \tau) \epsilon(0)=\frac{i N^{a}(0)}{4 \pi v\left(\tau-i x / v+\alpha \sigma_{\tau}\right)}, \\
& J_{L}^{a}(x, \tau) \epsilon(0)=\frac{-i N^{a}(0)}{4 \pi v\left(\tau+i x / v+\alpha \sigma_{\tau}\right)} .
\end{aligned}
$$

Observe that Eqs. 25)-(28) form a closed operator algebra - this is the key technical reason behind the generation of the inter-chain interaction of staggered magnetizations in frustrated spin chains models, see Ref. 15 and Sec. IIIB below.

\section{LOW-ENERGY HAMILTONIAN}

The spatially-anisotropic $J_{1}-J_{2}$ mode ${ }^{15}$ has taught us that keeping track of the nominally irrelevant terms is crucial for a correct solution of the problem. In this section, we extend this line of thinking to the crossed-chains model and demonstrate that indeed irrelevant terms produce symmetry-allowed relevant ones in a simple perturbation theory.

\section{A. Symmetry analysis}

Before proceeding with microscopic calculations, it is instructive to write down most general form of the interchain Hamiltonian $\delta V$ which is allowed by symmetries of the crossed-chains lattice. The reason to do so is that, while many such terms will be absent in a naïve continuum limit of the original spin model, those which are "accidentally" missing (i.e. not prohibited by any symmetry) may be expected to be generated as a "quantum correction" (i.e. through a RG transformation) when naïvely irrelevant terms are taken into account. The necessary complete set of space group generators for this analysis, $T_{h}, P_{s h}, P_{L h}$, and $R_{\pi / 2}$, was introduced in Sec. IA

Naturally (as in any field theory), there are an infinite number of possible interactions, and since there are additionally an infinite number of chains, the multitude of potential terms is compounded. Physically, however, "pairwise" interactions involving fields on only two chains at a time are expected to be most important (interactions involving more chains simultaneously can be shown to occur only in higher order in $\left.J_{\times} / J\right)$. Such an inter-chain Hamiltonian naturally splits into the sum of $\delta V_{\times}$, which describes interactions between two crossing chains, and $\delta V_{\|}$, which includes interactions between parallel chains, $\delta V=\delta V_{\times}+\delta V_{\|}$. Within these chain-pair interactions, we narrow the search by considering the "most relevant" possibilities (ones involving the smallest number of the smallest scaling dimension primary fields $\vec{N}$ and $\epsilon$ and no derivatives). Since we are perturbing the decoupledchain system, the appropriate sense of "relevant" is that of the decoupled 1+1-dimensional critical theories. We find

$$
\delta V_{\times}=\sum_{n, m} a_{1}(-1)^{n+m} \epsilon_{h, m}(n a) \epsilon_{v, n}(m a)
$$

and

$$
\begin{aligned}
\delta V_{\|}=\sum_{n, m, l} \sum_{\nu=h, v} & {\left[a_{2}(l) \vec{N}_{\nu, m}(n a) \cdot \vec{N}_{\nu, m+l}(n a)\right.} \\
+ & \left.a_{3}(l) \epsilon_{\nu, m}(n a) \epsilon_{\nu, m+l}(n a)\right] .
\end{aligned}
$$

We note that in Eq. (30), an interaction is possible between parallel chains an arbitrary distance $l$ apart. From the point of view of the decoupled-chain fixed point, there is no notion (or effect in RG rescaling) of "distance" between chains, so all such terms are equally "relevant" in this point of view. One would expect, however, these terms (i.e. $\left.a_{2}(l), a_{3}(l)\right)$ to decay in magnitude with increasing $l$. 
It is straightforward to check that these terms and only these terms satisfy the symmetry requirements of the checkerboard lattice. First, the invariance of $\delta V_{\|}$is easy to establish, as it involves pairs of operators $\epsilon$ and $\vec{N}$ from like chains (i.e., horizontal-horizontal or verticalvertical). These transform identically under all operations, and invariance is trivially shown.

The crossed-chains term, $\delta V_{\times}$, is more involved. We sketch the arguments for its invariance. Rotation by $\pi / 2$ about a crossing is manifest, as the fields in Eq. (29) are drawn from a single such crossing. Unit translation along the $x$-direction makes $\epsilon_{h} \rightarrow-\epsilon_{h}$ while $\epsilon_{v}$ is obviously not affected. However, $(-1)^{n+m}$ also changes its sign, $(-1)^{n+m} \rightarrow(-1)^{n+1+m}$, so that $T_{h}\left(\delta V_{\times}\right)=\delta V_{\times}$. Reflection with respect to a site on a horizontal chain $P_{s h}$ preserves $\epsilon_{v}$ but does change sign of dimerization on every horizontal chain: $P_{s h}\left(\epsilon_{h}\right)=-\epsilon_{h}$. But at the same time $P_{s h}$ interchanges even and odd vertical chains, i.e., $P_{s h}\left((-1)^{n+m}\right)=-(-1)^{n+m}$. Thus $P_{s h}\left(\delta V_{\times}\right)=\delta V_{\times}$. Link parity $P_{L h}$ is simple since every $\epsilon$ is even under it. Moreover, since $P_{L h}$ is nothing but reflection with respect to, say, the vertical chain number $n$, the vertical chain with index $n+1$ then transforms into that with index $n-1$, etc. Hence, even and odd vertical chains are not interchanged by $P_{L h}$, and $(-1)^{n+m} \rightarrow(-1)^{n+m}$, showing the invariance under this final generator. Notice that the staggering factor $(-1)^{n+m}$ plays a very important role in this consideration - its presence makes the local interaction of staggered dimerizations possible.

One could wonder if $\delta V_{\times}$could similarly include a staggered product of magnetizations, $(-1)^{n+m} \vec{N}_{h} \cdot \vec{N}_{h}$, but this is prohibited by the $P_{L h}$ symmetry. We note that microscopically, such a term cannot be generated (see the following subsection for the mechanics of generation of the allowed terms) as a consequence of the identity $J^{a}(x, \tau) \epsilon\left(x, \tau^{\prime}\right)=0$ which follows from the OPE Eq. (28). The only symmetry-allowed combination of $\vec{N}$ 's that can show up in $\delta V_{\times}$is $\left(\vec{N}_{h} \cdot \vec{N}_{v}\right)^{2}$. Such a term does arise in the large- $S$ "order-from-disorder" calculations, see Ref. $[3$, but in the $S=1 / 2$ microscopic model it has scaling dimension 2 and is thus deemed irrelevant. Moreover, one can derive, using abelian bosonization, the OPE of two $\vec{N}$ fields at the same spatial point $x$ : $N^{a}(x, \tau) N^{b}(x, 0) \sim i \epsilon^{a b c} \operatorname{sign}(\tau) J^{c}(x, 0)$. This allows one to identify ${ }^{26}$ this biquadratic term with the dimension 2 scalar product of two spin currents on crossing chains [that is, the $g_{j j}$ term in Eq. (23)], $\left(\vec{N}_{h} \cdot \vec{N}_{v}\right)^{2} \rightarrow \vec{J}_{h} \cdot \vec{J}_{v}$.

Observe now that none of the symmetry-respecting terms in $\delta V_{\times}$and $\delta V_{\|}$are present in the naïve continuum limit of the theory Eq. (23). Below we show that secondorder perturbation theory in the inter-chain exchange $J_{\times}$generates $\delta V_{\times}$with coupling constant $a_{1} \sim J_{\times}^{2} / J$. Similar arguments show that $a_{2,3} \sim J_{\times}^{4} / J^{3}$. This is because in $J_{\times}^{2}$ order one generates terms involving a product of derivatives of $\vec{N}$ fields on parallel chains, $\sim J_{\times}^{2} \partial_{x} \vec{N}_{h} \cdot \partial_{x} \vec{N}_{h}$. Once these are present, one can follow calculations in Ref. 15 to find that both $a_{2}$ and $a_{3}$ terms in $\delta V_{\|}$are generated, but this happens only in the next, $\left(J_{\times}^{2}\right)^{2}=J_{\times}^{4}$, order of the perturbation expansion. Since, as we show below in Sec. IIIC the $\delta V_{\times}$contribution is relevant, it is sufficient to keep only the leading $a_{1}$ type terms - subleading $a_{2,3}$ ones are too small $\left(a_{2,3} / a_{1} \sim J_{\times}^{2} / J^{2} \ll 1\right)$ to change the outcome.

\section{B. On the importance of irrelevant terms}

Here we describe microscopic calculation of $\delta V_{\times}$. We begin by expanding the action in powers of $V$ Eq. (23). This generates a number of terms of which the most important ones involve products of spin currents and staggered magnetizations from the same chain (and with the same spatial coordinate - that is, all fields belong to the same crossing). Thus we pick out $g_{j j} g_{n n}$ and cross-terms from $g_{n j}^{2}$. These contributions can be written in the form

$$
\left.\sum_{a, b} \sum_{n, m}(-1)^{n+m} \int d \tau d \tau^{\prime} v_{\times}\left(x, n ; y, m ; \tau, \tau^{\prime}\right)\right|_{x=n a, y=m a}
$$

where

$$
\begin{aligned}
v_{\times}=g_{n j}^{2}[ & \partial_{x} N_{h, m}^{a}(x, \tau) J_{h, m}^{b}\left(x, \tau^{\prime}\right) J_{v, n}^{a}(y, \tau) \partial_{y} N_{v, n}^{b}\left(y, \tau^{\prime}\right) \\
& \left.+J_{h, m}^{a}(x, \tau) \partial_{x} N_{h, m}^{b}\left(x, \tau^{\prime}\right) \partial_{y} N_{v, n}^{a}(y, \tau) J_{v, n}^{b}\left(y, \tau^{\prime}\right)\right] \\
+ & g_{j j} g_{n n} J_{h, m}^{a}(x, \tau) \partial_{x} N_{h, m}^{b}\left(x, \tau^{\prime}\right) \\
& \quad \times J_{v, n}^{a}(y, \tau) \partial_{y} N_{v, n}^{b}\left(y, \tau^{\prime}\right) .
\end{aligned}
$$

Now apply the OPE Eq. (27) to the product of fields from the same chain and at the same spatial point $x$. For example,

$$
\begin{aligned}
J_{R}^{a}(x, \tau) \partial_{x} N^{b}\left(x, \tau^{\prime}\right) & =\lim _{x^{\prime} \rightarrow x} \partial_{x^{\prime}} J^{a}(x, \tau) N^{b}\left(x^{\prime}, \tau^{\prime}\right) \\
& =\frac{-i\left[i \epsilon^{a b c} N^{c}\left(x, \tau^{\prime}\right)-i \delta^{a b} \epsilon\left(x, \tau^{\prime}\right)\right]}{4 \pi v^{2}\left(\tau-\tau^{\prime}+\alpha \sigma_{\tau-\tau^{\prime}}\right)^{2}}
\end{aligned}
$$

Similarly

$$
J_{L}^{a}(x, \tau) \partial_{x} N^{b}\left(x, \tau^{\prime}\right)=\frac{i\left[i \epsilon^{a b c} N^{c}\left(x, \tau^{\prime}\right)+i \delta^{a b} \epsilon\left(x, \tau^{\prime}\right)\right]}{4 \pi v^{2}\left(\tau-\tau^{\prime}+\alpha \sigma_{\tau-\tau^{\prime}}\right)^{2}} .
$$

We observe that the OPE of the full spin current $\vec{J}$ and $\vec{N}$ at the same spatial point does not contain staggered magnetization

$$
\begin{aligned}
J^{a}(x, \tau) \partial_{x} N^{b}\left(x, \tau^{\prime}\right) & =\left[J_{R}^{a}(x, \tau)+J_{L}^{a}(x, \tau)\right] \partial_{x} N^{b}\left(x, \tau^{\prime}\right) \\
& =-\frac{\delta^{a b} \epsilon\left(x, \tau^{\prime}\right)}{2 \pi v^{2}\left(\tau-\tau^{\prime}+\alpha \sigma_{\tau-\tau^{\prime}}\right)^{2}} .
\end{aligned}
$$

This is a very important result, with which Eq. 31] can be brought into the surprisingly compact form:

$$
\begin{aligned}
V_{2}= & \sum_{a, b} \delta^{a b} \sum_{n, m}(-1)^{n+m}\left(2 g_{n j}^{2}+g_{j j} g_{n n}\right) \\
& \times \int d \tau d \tau^{\prime} \frac{\epsilon_{h, m}\left(n a, \tau^{\prime}\right) \epsilon_{v, n}\left(m a, \tau^{\prime}\right)}{\left[2 \pi v^{2}\left(\tau-\tau^{\prime}+\alpha \sigma_{\tau-\tau^{\prime}}\right)^{2}\right]^{2}}
\end{aligned}
$$


The integral involved is obviously convergent

$$
\int_{-\infty}^{\infty} d t \frac{1}{\left(t+\alpha \sigma_{t}\right)^{4}}=\frac{2}{3 \alpha^{3}}=\frac{2 v^{3}}{3 a^{3}} .
$$

Using Eq. (24) for the $g$ 's involved, we finally obtain the following fluctuation-generated correction to the lowenergy effective action

$$
\begin{aligned}
\delta S & =-\frac{1}{2} V_{2} \\
& =-\frac{3 J_{\times}^{2} a^{3}}{\pi^{2} v} \int d \tau \sum_{n, m}(-1)^{n+m} \epsilon_{h, m}(n a, \tau) \epsilon_{v, n}(m a, \tau) .
\end{aligned}
$$

Denoting

$$
g_{\epsilon}=v \frac{3 J_{\times}^{2} a^{2}}{\pi^{2} v^{2}}
$$

we have the following addition to the inter-chain Hamiltonian $V$, Eq. (23), to analyze [this is because $Z=\int e^{-S}$ and $\left.\delta S=\int d \tau \delta V\right]$

$$
\delta V_{\times}=-g_{\epsilon} a \sum_{n, m}(-1)^{n+m} \epsilon_{h, m}(n a) \epsilon_{v, n}(m a) .
$$

Staggered dimerization $\epsilon$ has scaling dimension $1 / 2$, which means that it is as important for the chain physics as $\vec{N}$ is. In fact, up to logarithmic corrections, correlation functions of staggered dimerization and magnetization decay with the same power law, $x^{-1}$, see (12).

This is also clear from the OPE Eqs. (27) and (28), which show that $\vec{N}$ and $\epsilon$ transform into each other under chiral rotations generated by $\vec{J}_{R / L}$. Since any pair of horizontal and vertical chains has only one crossing, Eq. (40) is a sum of local terms, each of which is marginal (space-time dimension $=1$, and dimension of the product $\epsilon_{h} \epsilon_{v}$ is 1 as well). However, we shall see below that this marginality is superficial - an infinite number of marginal crossings add up to a relevant perturbation.

\section{Mean-field analysis of the effective inter-chain interaction Eq. (40)}

From now on it is safe to omit derivative terms present in $V$, Eq. [23]; their role was to generate, as described in Sec.IIIB more relevant symmetry-allowed inter-chain interactions. With this in mind, we write down the renormalized version of Eq. (23),

$$
\begin{aligned}
V=\sum_{n, m}[ & g_{j j} \vec{J}_{h, m}(n a) \cdot \vec{J}_{v, n}(m a) \\
& \left.\quad-(-1)^{n+m} g_{\epsilon} a \epsilon_{h, m}(n a) \epsilon_{v, n}(m a)\right] .
\end{aligned}
$$

As discussed above, the first term originating from the naive continuum limit of Eq. (8) has scaling dimension 2 while the second term, which is $\delta V_{\times}$generated by highenergy fluctuations, has scaling dimension 1 . Thus we are allowed to discard the irrelevant current-current piece of $V, \mathrm{Eq}$. 41). As a result, all that remains of the interchain interaction is given by $\delta V_{\times}$[Eq. [40] $], V \rightarrow \delta V_{\times}$, which was not present in the naive continuum limit Eq. (23) at all! We tackle it, in analogy with analysis of Ref. 15, by the chain mean-field approximation. The staggering factor $(-1)^{n+m}$ suggests a staggered dimer order on parallel chains. That is, we assume the pattern

$$
\epsilon_{h, m}(x)=(-1)^{m}\langle\epsilon\rangle, \quad \epsilon_{v, n}(y)=(-1)^{n}\langle\epsilon\rangle,
$$

where $\langle\epsilon\rangle$ is a mean-field expectation value. The interchain coupling is then decoupled into a sum of independent single-chain Hamiltonians,

$$
\begin{aligned}
\delta V_{\times}= & -\sum_{m}(-1)^{m} g_{\epsilon} a\langle\epsilon\rangle \sum_{n} \epsilon_{h, m}(x=n a) \\
& -\sum_{n}(-1)^{n} g_{\epsilon} a\langle\epsilon\rangle \sum_{m} \epsilon_{v, n}(y=m a) .
\end{aligned}
$$

Look on one of them, say, that of the horizontal chain with index $m$ (which is fixed now). Now we can take continuum limit $\left(\sum_{n} f(n a) \rightarrow a^{-1} \int d x f(x)\right)$ and

$$
\delta V_{\times}(m)=-(-1)^{m} g_{\epsilon}\langle\epsilon\rangle \int \epsilon(x) d x,
$$

which can be easily analyzed along the lines of Ref. 15. Using abelian bosonization expression for the staggered dimerization

$$
\epsilon(x)=\frac{\lambda}{\pi a} \cos [\sqrt{2 \pi} \varphi(x)],
$$

where $\varphi$ is the spin boson field and $\lambda$ is a nonuniversal constant of order 1,27 we arrive at the effective singlechain sine-Gordon action for the $m$ th chain

$$
S(m)=\int d^{2} r\left(\frac{1}{2}(\nabla \varphi)^{2}-G \cos \sqrt{2 \pi} \varphi\right) .
$$

The action $S(m)$ is written in terms of dimensionless coordinates $\vec{r}=(x / a, v \tau / a)$ and the effective coupling constant $G=\lambda^{2} g_{\epsilon}\langle\cos \sqrt{2 \pi} \varphi\rangle /\left(\pi^{2} v\right)$. The self-consistent equation for $\langle\cos \sqrt{2 \pi} \varphi\rangle$ follows from the exact solution 28 for the free energy $F_{m}$ of the sine-Gordon model $Z_{m}=$ $\int D \varphi \exp [-S(m)]=\exp \left(-F_{m}\right)$;

$$
\langle\cos \sqrt{2 \pi} \varphi\rangle=-\frac{d F}{d G}=\frac{d \ln Z}{d G}=\frac{c_{0}^{2}}{3 \sqrt{3}} G^{1 / 3},
$$

where the constant $c_{0}$ reads

$$
c_{0}=\frac{2 \Gamma(1 / 6)}{\sqrt{\pi} \Gamma(2 / 3)}\left(\frac{\pi \Gamma(3 / 4)}{2 \Gamma(1 / 4)}\right)^{2 / 3} .
$$

Simple algebra gives

$$
\langle\cos \sqrt{2 \pi} \varphi\rangle=\frac{c_{0}^{3}}{3} \sqrt{\frac{\lambda^{2} g_{\epsilon}}{\pi^{2} v}}=0.265316 \frac{J_{\times}}{J},
$$


where we have set $\lambda=1$. Hence the expectation value of the staggered dimerization is proportional to $J_{\times}$,

$$
\langle\epsilon\rangle=\frac{\lambda}{\pi a}\langle\cos \sqrt{2 \pi} \varphi\rangle=0.0844 \frac{J_{\times}}{J a} .
$$

The spin gap $\Delta$ is given by the mass $m$ of the lightest breather in the sine-Gordon theory ${ }^{28}$

$$
m=2 M \sin (\pi / 6)=M,
$$

where $M$ and $G$ are related by

$$
\frac{G}{2}=\frac{\Gamma(1 / 4)}{\pi \Gamma(3 / 4)}\left(M \frac{\sqrt{\pi} \Gamma(2 / 3)}{2 \Gamma(1 / 6)}\right)^{3 / 2} .
$$

Thus $M=c_{0} G^{2 / 3}$, and finally the spin gap $\Delta$ is found as

$$
\Delta=m=M=\frac{4 \lambda^{2} c_{0}^{3}}{\pi^{6} \sqrt{3}}\left(\frac{J_{\times}}{J}\right)^{2}=0.675688\left(\frac{J_{\times}}{J}\right)^{2} .
$$

The resulting dimerization pattern is shown in Fig. 2 An equivalent configuration is obtained by a global shift of crosses by one lattice spacing along either the $x$ or $y$ direction. It is worth pointing out that exactly such inter-dimer correlations - crossed-dimer ones - have been observed in the exact diagonalization study of finite CCM clusters, see Table II and Fig. 5 in Ref. 11.

\section{THE PLANAR PYROCHLORE: PLAQUETTE VBS AND ITS INSTABILITIES}

In the preceding sections we focused on the quasi-1D limit $J_{\times} \ll J$, and established the existence of the spontaneous long-range order of the crossed dimer configuration (Fig. 22). In this section we will explore a different region in the parameter space, where the nearest-neighbor coupling $J_{\times}$and next-nearest-neighbor exchange coupling $J$ are nearly equal. Earlier numerical studies using exact diagonalization 10 and strong-coupling expansion techniques 12.13 .14 showed that the ground state at $J=$ $J_{\times}$is a valence bond crystal with long-range quadrumer order, shown in Fig. [3 Here we review a simple theoretical account of this plaquette VBS (P-VBS) state using the quadrumer-boson approximation, 29.30 .31 and examine its instabilities to other orders. This analysis, together with the results in the preceding sections, will serve as a basis for our discussion on the global phase diagram of the CCM in the following section. Our simple approach presented here is meant to give a qualitative picture; more quantitatively reliable numerical results can be obtained, for example, by series expansion, as developed in Refs. 12 and 14 .

In the following analysis it is more convenient to use a new coordinate system labelled by $(j, k)$, rotated by $\pi / 4$ from the $x$ and $y$ axes; see Fig. 3 . The quadrumerized valence bond crystal breaks lattice translation symmetry,

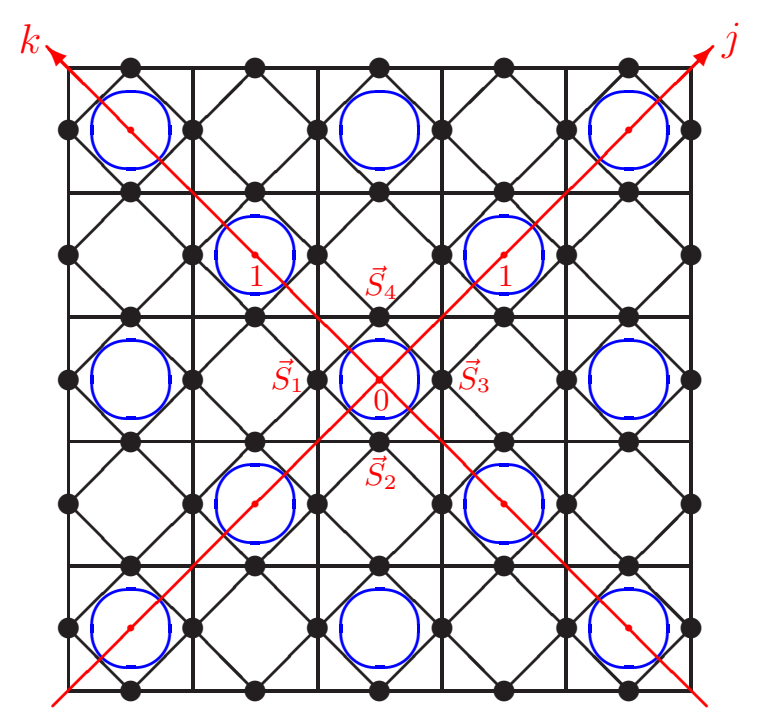

FIG. 3: (Color online) Quadrumerized checkerboard lattice with coordinates $(j, k)$ shown. The plaquettes with blue circles are quadrumerized. Each unit cell contains 4 spins.

and each quadrumerized plaquette centered at $(j, k)$ has four spins, $\vec{S}_{l}(l=1,2,3,4)$. From the outset we assume the breaking of translation symmetry and begin with the Hamiltonian for a single quadrumerized plaquette,

$$
\begin{aligned}
H_{p}=J_{\times} & \left(\vec{S}_{1} \cdot \vec{S}_{2}+\vec{S}_{2} \cdot \vec{S}_{3}+\vec{S}_{3} \cdot \vec{S}_{4}+\vec{S}_{4} \cdot \vec{S}_{1}\right) \\
=\frac{J_{\times}}{2} & {\left[\left(\vec{S}_{1}+\vec{S}_{2}+\vec{S}_{3}+\vec{S}_{4}\right)^{2}-\left(\vec{S}_{1}+\vec{S}_{3}\right)^{2}\right.} \\
& \left.-\left(\vec{S}_{2}+\vec{S}_{4}\right)^{2}\right] .
\end{aligned}
$$

The lowest-energy state of $H_{p}$ is a spin singlet with energy $-2 J_{\times}$, which can be written as

$$
\begin{gathered}
s^{\dagger}|0\rangle=\frac{1}{2 \sqrt{3}}(|\uparrow \uparrow \downarrow \downarrow\rangle+|\downarrow \downarrow \uparrow \uparrow\rangle+|\uparrow \downarrow \downarrow \uparrow\rangle+|\downarrow \uparrow \uparrow \downarrow\rangle \\
-2|\uparrow \downarrow \uparrow \downarrow\rangle-2|\downarrow \uparrow \downarrow \uparrow\rangle),
\end{gathered}
$$

where $\left|\sigma_{1} \sigma_{2} \sigma_{3} \sigma_{4}\right\rangle$ denotes the state with $S_{l}^{z}=\sigma_{l}$. The first excited states are a triplet with energy $-J_{\times}$,

$$
\begin{aligned}
t_{+}^{\dagger}|0\rangle & =\frac{1}{2}(|\uparrow \uparrow \uparrow \downarrow\rangle+|\uparrow \downarrow \uparrow \uparrow\rangle-|\uparrow \uparrow \downarrow \uparrow\rangle-|\downarrow \uparrow \uparrow \uparrow\rangle), \\
t_{z}^{\dagger}|0\rangle & =\frac{1}{\sqrt{2}}(|\uparrow \downarrow \uparrow \downarrow\rangle-|\downarrow \uparrow \downarrow \uparrow\rangle), \\
t_{-}^{\dagger}|0\rangle & =\frac{1}{2}(|\downarrow \downarrow \downarrow \uparrow\rangle+|\downarrow \uparrow \downarrow \downarrow\rangle-|\downarrow \downarrow \uparrow \downarrow\rangle-|\uparrow \downarrow \downarrow \downarrow\rangle) .
\end{aligned}
$$

The operators $s^{\dagger}, t_{ \pm}^{\dagger}$, and $t_{z}^{\dagger}$ can be thought of as creation operators of hard-core bosons.

As mentioned above, the ground state of the CCM is known to be a gapped P-VBS state at the planar pyrochlore point $J=J_{\times}$. As long as $J \approx J_{\times}$, we may 
thus expect that a good approximation to the ground state should be obtained by direct product of the singlet states, Eq. (55), weakly hybridized with the triplets, Eqs. (56). Motivated by this observation, we employ the quadrumer-boson approximation ${ }^{29.30 .31}$ in which we keep only the low-lying four states, singlet and triplet, in each quadrumerized plaquette, and discard the other higherenergy states. Now the boson operators are subject to the constraint

$$
s^{\dagger} s+t_{+}^{\dagger} t_{+}+t_{z}^{\dagger} t_{z}+t_{-}^{\dagger} t_{-}=1
$$

The plaquette Hamiltonian is then written as

$$
H_{p}=-2 J_{\times}+J_{\times}\left(t_{+}^{\dagger} t_{+}+t_{z}^{\dagger} t_{z}+t_{-}^{\dagger} t_{-}\right) .
$$

The spins $\vec{S}_{l}$ can also be written in terms of the hard-core boson operators. The representations are found from matrix elements of the spin operators with the four states. After some algebra we find

$$
\begin{aligned}
& S_{l}^{z}=\frac{1}{4}\left(t_{+}^{\dagger} t_{+}-t_{-}^{\dagger} t_{-}\right)+\frac{(-1)^{l}}{\sqrt{6}}\left(t_{z}^{\dagger} s+s^{\dagger} t_{z}\right), \\
& S_{l}^{+}=\frac{1}{\sqrt{8}}\left(t_{+}^{\dagger} t_{z}-t_{z}^{\dagger} t_{-}\right)-\frac{(-1)^{l}}{\sqrt{3}}\left(t_{+}^{\dagger} s+s^{\dagger} t_{-}\right), \\
& S_{l}^{-}=\frac{1}{\sqrt{8}}\left(t_{z}^{\dagger} t_{+}-t_{-}^{\dagger} t_{z}\right)-\frac{(-1)^{l}}{\sqrt{3}}\left(t_{-}^{\dagger} s+s^{\dagger} t_{+}\right),
\end{aligned}
$$

where $l=1,2,3,4$. Assuming that the density of triplets is low in the P-VBS state, we keep only terms linear in $t_{\mu}$ and set $s=s^{\dagger}=1$;

$$
S_{l}^{a}=\frac{(-1)^{l}}{\sqrt{6}}\left(t_{a}^{\dagger}+t_{a}\right)
$$

where $a=x, y, z$ and we have introduced

$$
t_{x}=-\frac{1}{\sqrt{2}}\left(t_{+}+t_{-}\right), \quad t_{y}=\frac{1}{\sqrt{2} i}\left(t_{+}-t_{-}\right) .
$$

With the coordinate system $(j, k)$ in Fig. 3 the total Hamiltonian of the checkerboard antiferromagnet reads

$$
\begin{aligned}
& H=\sum_{j, k} H_{p}(j, k) \\
&+J_{\times} \sum_{j, k}\left(\vec{S}_{j, k, 1} \cdot \vec{S}_{j-1, k, 4}+\vec{S}_{j, k, 2} \cdot \vec{S}_{j, k-1,1}\right. \\
&\left.\quad+\vec{S}_{j, k, 3} \cdot \vec{S}_{j+1, k, 2}+\vec{S}_{j, k, 4} \cdot \vec{S}_{j, k+1,3}\right) \\
&+J \sum_{j, k}\left(\vec{S}_{j, k, 1} \cdot \vec{S}_{j, k+1,3}+\vec{S}_{j, k, 4} \cdot \vec{S}_{j, k+1,2}\right. \\
&\left.\quad+\vec{S}_{j, k, 3} \cdot \vec{S}_{j+1, k, 1}+\vec{S}_{j, k, 4} \cdot \vec{S}_{j+1, k, 2}\right)
\end{aligned}
$$

where $\vec{S}_{j, k, l}$ is the $l$ th spin $\vec{S}_{l}$ in the quadrumerized plaquette centered at $(j, k)$. With the approximation
Eq. (60) the Hamiltonian becomes

$$
H=\sum_{\vec{p}}\left(-\frac{7}{2} J_{\times}+\frac{1}{2} \sum_{a=x, y, z} \Psi_{a}^{\dagger}(\vec{p}) \mathcal{H}(\vec{p}) \Psi_{a}(\vec{p})\right),
$$

where we have introduced the triplet boson field,

$$
\Psi_{a}(\vec{p})=\left(\begin{array}{c}
\tilde{t}_{a}(\vec{p}) \\
\tilde{t}_{a}^{\dagger}(-\vec{p})
\end{array}\right),
$$

with the momentum $\vec{p}=\left(p_{1}, p_{2}\right)$ and the Fourier transform

$$
\tilde{t}_{a}(\vec{p})=\frac{1}{\sqrt{\mathcal{N}_{\circ}}} \sum_{j, k} e^{-i\left(j p_{1}+k p_{2}\right)} t_{a}(j, k),
$$

where $\mathcal{N}_{\circ}$ is the number of quadrumerized plaquettes. The Hamiltonian matrix is given by

$$
\mathcal{H}(\vec{p})=\left(\begin{array}{cc}
J_{\times}+\varepsilon(\vec{p}) & \varepsilon(\vec{p}) \\
\varepsilon(\vec{p}) & J_{\times}+\varepsilon(\vec{p})
\end{array}\right),
$$

where

$$
\varepsilon(\vec{p})=\frac{2}{3}\left(J-J_{\times}\right)\left(\cos p_{1}+\cos p_{2}\right) .
$$

With the Bogoliubov transformation,

$$
\left(\begin{array}{c}
\tilde{t}_{a}(\vec{p}) \\
\tilde{t}_{a}^{\dagger}(-\vec{p})
\end{array}\right)=\left(\begin{array}{cc}
\cosh \theta_{\vec{p}} & \sinh \theta_{\vec{p}} \\
\sinh \theta_{\vec{p}} & \cosh \theta_{\vec{p}}
\end{array}\right)\left(\begin{array}{c}
b_{a}(\vec{p}) \\
b_{a}^{\dagger}(-\vec{p})
\end{array}\right),
$$

where

$$
\exp \left(-4 \theta_{\vec{p}}\right)=1+\frac{2 \varepsilon(\vec{p})}{J_{\times}}
$$

the Hamiltonian [Eq. [63] ] is diagonalized,

$$
H=\sum_{\vec{p}}\left(-\frac{7}{2} J_{\times}+\frac{3}{2} E(\vec{p})+E(\vec{p}) \sum_{a} b_{a}^{\dagger}(\vec{p}) b_{a}(\vec{p})\right) .
$$

The energy dispersion of the triplet eigen mode $b_{a}(\vec{p})$ is given by

$$
E(\vec{p})=\left[J_{\times}\left(J_{\times}+\frac{4}{3}\left(J-J_{\times}\right)\left(\cos p_{1}+\cos p_{2}\right)\right)\right]^{1 / 2} .
$$

The simple quadrumer-boson approximation described above reproduces the basic feature of the previous numerical studies: $10,12,13,14$ at $J \approx J_{\times}$the P-VBS state is a stable singlet ground state with a gap to excited states.

The softening of the triplet mode Eq. (71) tells us potential instabilities that the P-VBS state may have (see also Ref. 14). First, from $E(0)=\left[J_{\times}\left(8 J-5 J_{\times}\right) / 3\right]^{1 / 2}$ we see that it becomes unstable at $J_{\times} \rightarrow 8 J / 5$, when the bosons condense at $\vec{p}=0$. The resulting state has the long-range Néel order, like the one realized in 


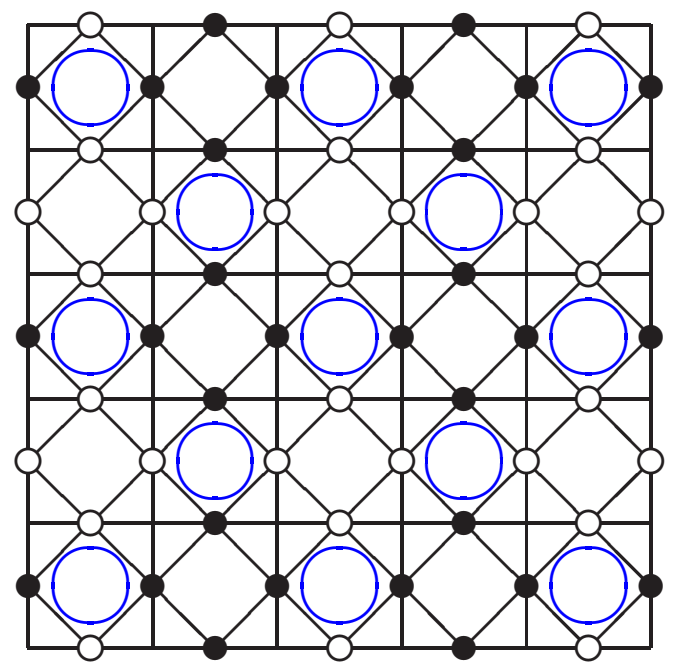

FIG. 4: (Color online) The magnetically ordered state due to condensation of bosons $b_{a}(\pi, \pi)$. The solid circles represent, say, up spins, and the open circles represent down spins. The quadrumerized plaquettes in the nearby P-VBS phase are indicated by blue circles.

the square-lattice limit $J_{\times} \gg J$. This can be easily seen from Eq. [60), in which we may suppose that $t_{a}^{\dagger}+t_{a}$ is a nonvanishing $c$-number upon condensation of bosons. In the present approximation, the transition from the plaquette singlet to the Néel-ordered state occurs at $\left(J / J_{\times}\right)_{c 1}=5 / 8$, which should be compared with the estimate $\left(J / J_{\times}\right)_{c 1}=0.79 \sim 0.81$ from a sophisticated strong-coupling expansion 14 Second, the P-VBS state becomes unstable as $J / J_{\times} \rightarrow\left(J / J_{\times}\right)_{c 2}=11 / 8$, at which bosons with momentum $\vec{p}=(\pi, \pi)$ condense. Upon bose condensation the spins will have a magnetic long-range order, shown in Fig. 团 with the spin configuration $\uparrow \uparrow \downarrow \downarrow \uparrow \uparrow \downarrow \downarrow$ along the diagonal directions and the Néel order along the horizontal and vertical chain directions [as can be seen by replacing $t_{a}^{\dagger}+t_{a} \rightarrow(-1)^{j+k}$ in Eq. [60)]. In fact, this is one of the magnetically ordered states which are shown to be stable at $J \gg J_{\times}$in the $1 / S$ expansion; see Fig. 2(b) and Fig. 6 in Ref. 3. The softening of the triplet mode at $\vec{p}=(\pi, \pi)$ is also found in the strong-coupling expansion in Ref. 14 with the estimated critical coupling $\left(J / J_{\times}\right)_{c 2}=1.06 \sim 1.13$.

Finally, we comment on two defects in our approach. One is that the lattice translation symmetry is explicitly broken from the outset and cannot be restored within the theory. This means that the Néel ordered state at $J_{\times}>5 J / 8$ inevitably has the P-VBS order as well i.e., it is a coexistence phase. This should probably be viewed as an artifact of the approach - see Sec. $\nabla$ for discussion of this portion of the phase diagram. The other defect is that we have ignored interactions among the triplet bosons (as well as projected out the other higher-

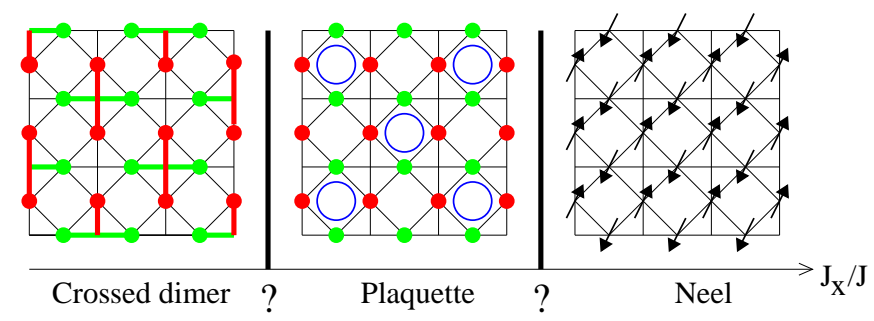

FIG. 5: (Color online) Global phase diagram of the CCM model in scenario I of Sec. VA Thick vertical lines with question marks indicate that the corresponding transition is, according to Landau theory reasoning, either first-order or occurs via an intermediate co-existence phase.

energy states - singlet, triplet, and quintuplet - on each quadrumerized plaquette). In the crudest approximation we adopted, the first excited states are a triplet excitation $S=1$ with no energy dispersion at $J_{\times}=J$. The numerical studies 10.12.13.14 showed, however, that at $J=J_{\times}$the lowest-excited states are in the spin singlet sector (see, for example, Sec. V in Ref. 10). The cluster-based calculations ${ }^{12.13}$ indicate that these singlet excitations are bound states of two $S=1$ excitations. To describe correctly the singlet excitations in terms of the quadrumer bosons, one needs to go beyond the linear approximation Eq. (60) and include interactions among the bosons. We do not try to do this here, but only point out that the dispersionless triplet mode is naturally susceptible to forming a bound state. Away from the planar pyrochlore point $J_{\times}=J$, not much is known about the low-energy excitations in the $S=0$ sector; it is not well understood how the singlet energy levels in the spin gap change as a function of $J_{\times} / J^{32}$

\section{GLOBAL PHASE DIAGRAM OF THE CCM}

In this section we discuss the global zero-temperature phase diagram of the CCM as the control parameter $J_{\times} / J$ is increased from 0 to $\infty$. Our analysis relies on three well-established facts. First, the ground state at $J_{\times} \gg J$ obviously has the Néel order and is smoothly connected to the Néel ordered state of the antiferromagnetic Heisenberg model on the square lattice. Second, by now there is convincing numerical10,12,13.14 and analytical 4.5 .6 evidence for the P-VBS state at and around $J=J_{\times}$. Finally, as shown in Sec. III the ground state is spontaneously dimerized in the quasione-dimensional $J_{\times} \ll J$ limit as well, where long-range crossed-dimer order (Fig. 21) sets in.

The first question we address here is how the two dimerized phases, plaquette VBS (P-VBS) and crosseddimer VBS (CD-VBS), are connected. We propose the two complementary scenarios in subsections $\mathrm{VA}$ and $\mathrm{VB}$ below. The nature of the transition between the P-VBS and the Néel states is discussed in Sec. VC 


\section{A. Scenario I: direct transition between the crossed-dimer and plaquette VBS}

The 'minimal' assumption is that the two quantumdisordered valence bond phases connect at some critical value $J_{\times} / J<1$. The validity of this assumption can only be verified by the exact calculation of the ground state energies of two dimerized phases in the whole interval $0<J_{\times} / J<1$ of interest. Such a calculation is obviously beyond our analytic approximations suited for $J_{\times} / J \rightarrow 0$ (CD-VBS, Sec. IIII) and $J_{\times} / J \rightarrow 1$ (P-VBS, Sec. IIV] limits. Instead, we take a phenomenological point of view here and assume that the ground state energies are such that a direct transition between the CD-VBS and P-VBS phases is possible. At least partial support to this point of view is provided by the exact diagonalization study of Sindzingre et $a l^{11}$ which seems to indicate a single change in the ground state around $J_{\times} / J \approx 0.8$.

The question then is if this transition between these two phases can be continuous. In general, this question is difficult to answer. Most formally, the renormalization group theory of continuous critical phenomena sets only some rather weak constraints on the existence of a continuous phase transition between any two phases "A" and "B". In particular, it requires the existence of an abstract scale-invariant fixed point (critical field theory) with a single "relevant" symmetry-allowed operator in its spectrum, such that a positive/negative coefficient of this operator in the action takes the system into phase A/B. The critical fixed point theory itself must clearly have higher symmetry than either phase A or B, but no a priori restriction is placed on the relation of the symmetries of phase A to those of phase B.

A conventional - and more stringent - "criterion" for the existence of a continuous transition is based on the specific realization of the critical theory provided by a Landau-Ginzburg-Wilson (LGW) action written in terms of order parameters. More physically, LGW theory permits a continuous transition by the "condensation" of some "soft mode" of phase A, which transforms nontrivially under the symmetry group of A. The condensation of this soft-mode order parameter then leads to a lowering of symmetry (since by assumption the condensation breaks some symmetries that it transforms under) in phase B. A necessary criterion for a LGW-allowed continuous phase transition is thus that the symmetry group of one phase ( $\mathrm{B}$ in the example) is a sub-group of the other (phase A) 33 Further restrictions are implied by detailed consideration of the LGW expansion (e.g., presence of cubic terms, etc.), as is standard 33

Recent work on related but distinct quantum phase transitions has provided explicit theoretical examples of non-LGW critical theories, 34 demonstrating that the violation of this conventional "criterion" is more than a formal possibility. Unfortunately, there is at present no general prescription to supplant the LGW criterion, so we are left in the uncomfortable position of being unable to solidly argue for or against the possibility of a con-

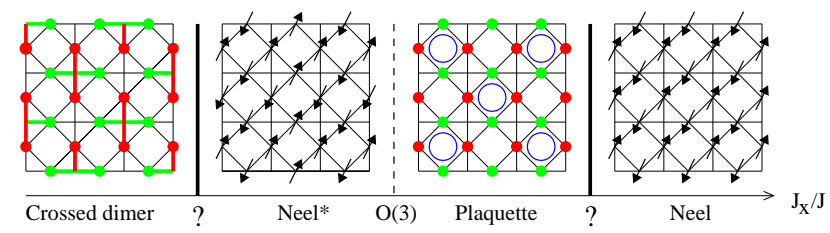

FIG. 6: (Color online) Global phase diagram of the CCM model in scenario II of Sec. VB The continuous $O(3)$ transition between the Néel ${ }^{*}$ and P-VBS phases is indicated by a dashed vertical line. Other notations are as in Fig. 15

tinuous quantum critical point (QCP). Instead, we will content ourselves here with the LGW analysis.

It is straightforward to conclude that a continuous transition between the CD-VBS and P-VBS states is prohibited by the LGW criterion. This can be seen by the two lattice reflections $\mathcal{R}_{1,2}$, which map the crossedchains lattice onto itself, i.e., symmetries of the Hamiltonian. Here $\mathcal{R}_{1}$ is the reflection with respect to a horizontal chain [it corresponds to a link parity operation $P_{L}$ Eq. (18) on all vertical chains], and $\mathcal{R}_{2}$ is the reflection with respect to a horizontal line passing through the centers of empty plaquettes [this is a site parity $P_{s}$ Eq. (17) from the point of view of vertical chains]; similar reflections with respect to vertical lines are accounted for by the $\pi / 2$ rotational symmetry (about chain crossings) of the lattice.

Both phases are two-fold degenerate, as can been seen from Fig. [5] (and hence can be described by Ising order parameters). Their symmetries are distinct. In particular, note first that $\mathcal{R}_{1}$ is a symmetry of the CD-VBS phase, but not the P-VBS (it interchanges the two PVBS ground states). Thus the symmetry group of the CD-VBS phase is not a subgroup of that of the P-VBS phase. Second, $\mathcal{R}_{2}$ is a symmetry of the P-VBS phase, but not the CD-VBS phase. Thus the symmetry group of the $\mathrm{P}-\mathrm{VBS}$ phase is not a subgroup of the CD-VBS state. Since neither symmetry group is a subgroup of the other, a continuous LGW transition between the two states is not possible, as promised.

The simplest alternative is a first order transition between the two phases, which is always possible, and may perhaps be likely. Another possibility, is that, between the two states, there is a finite range of coexistence of $\mathrm{P}$ VBS and CD-VBS order. Such a coexistence phase can have continuous LGW (Ising) transitions to both the PVBS and CD-VBS states. The latter scenario is only one of a multitude of conceptually possible phase structures, for which we have no physical motivation. We indicate this uncertainty by the question mark in Fig. 5

\section{B. Scenario II: CD to P-VBS via an intermediate ordered phase}

A quite different scenario is suggested by the quadrumer-boson approximation of Sec. [IV an interme- 
diate magnetically ordered phase between the CD-VBS and P-VBS states. It was found there that the P-VBS state becomes unstable at $\left(J_{\times} / J\right)_{c 2}=8 / 11$ as the $J_{\times} / J$ ratio is reduced below the planar pyrochlore value of 1 . The resulting state, depicted in Fig. 4 and Fig. 6] possesses long-range magnetic order. We denote it as the Néel* state in the following. This magnetically ordered state was previously found in the large- $S$ approach ${ }^{3}$, where it arises as a result of a quantum "order-fromdisorder" selection among the large family of degenerate (at $S=\infty$ ) collinear ordered states. The fact that it also appears as a result of the triplet softening of the $S=1 / 2$ $\mathrm{P}-\mathrm{VBS}$ phase of Sec. IV gives a strong independent argument in favor of its stability. Of course, as in the previous scenario, the ultimate fate of the Néel* phase is to be decided by accurate numerical investigations, and here we just assume that this ordered phase is indeed the ground state in a finite $J_{\times} / J$ window within the $(0,1)$ interval.

The transition between the P-VBS and the Néel* phases manifests itself as a triplet condensation transition within the consideration of Sec. IV Since such a "soft-mode" transition is the general physical interpretation of LGW theory, it should not come as a surprise that the Néel* to P-VBS transition satisfies the LGW criterion for a continuous critical point. In particular, the symmetry group of the Néel $^{*}$ state is generated by: (1) spin rotations about the ordering axis, (2) translations along a diagonal $[(1,1)$ or $(1,-1)$ in the coordinate system of Fig. 6] composed with a time-reversal which inverts the spins $\vec{S}_{r} \rightarrow-\vec{S}_{r}$, (3) reflections $\mathcal{R}_{2}$, and (4) $\pi / 2$ rotations about the center of a plaquette containing four parallel spins. Plainly, from Fig. 6] every one of these operations is also a symmetry of the P-VBS state; hence the symmetry group of the Néel* state is a subgroup of that of the P-VBS state. Moreover, the triplet condensation amplitude can be identified with the Néel* order parameter: an $O(3)$ vector specifying the direction of spin orientation at some reference site in Fig. 6] Indeed, an LGW expansion could be developed in this order parameter, but we content ourselves with the expectation that the P-VBS to Néel* transition is likely in the continuous $O(3)$ universality class.

Clearly, the Néel* phase cannot survive down to the $J_{\times} / J=0$ point which describes decoupled $S=1 / 2$ spin chains, as quantum spin fluctuations destroy antiferromagnetic Néel long-range order in individual chains. ${ }^{3}$ The calculations of Sec. III demonstrate that at small $J_{\times} / J$, the fluctuating dimerization field $\epsilon$ takes over the (quasi-classical) spin fluctuations and drives the chains into the CD-VBS phase.

We can again apply the LGW criterion to ask whether a continuous CD-VBS to Néel* transition is possible. Clearly, the symmetry group of the CD-VBS state cannot be a subgroup of the Néel* phase, since the former is spin-rotationally invariant. However, the Néel* phase is invariant under $\mathcal{R}_{2}$, which, as we saw in the previous subsection, is not a symmetry of the CD-VBS phase. Thus, the symmetry group of the Néel* state is not a subgroup of that of the CD-VBS phase, and a continuous LGW transition between these two phases is prohibited.

The transition then is likely either a first-order one or proceeds via an intermediate ordered and bondmodulated coexistence phase. Such a phase is easy to imagine — start with the Néel* state and modulate slightly spin exchange couplings along horizontal and vertical chains so that "strong" bonds repeat the pattern of dimers in the CD-VBS phase. This state clearly breaks $\mathcal{R}_{2}$, but does preserve the long-range magnetic order (for sufficiently weak modulation) of the Néel* phase. The transition from the CD-VBS to such a "modulated" Néel* one is then continuous $O(3)$ spin-symmetry breaking, while at some higher $J_{\times} / J$ the bond modulation goes away via a continuous $Z_{2}$ transition and one obtains the pure Néel* phase. Which of the two possibilities, firstorder or coexistence, is realized cannot be decided within our analytical approach, and for this reason the transition between CD-VBS and Néel* phases is marked by a question mark in Fig. 6]

\section{Plaquette VBS to Néel transition}

Regardless of the phase structure for $J_{\times} / J<1$, we expect a transition at larger $J_{\times}$from the P-VBS state to the conventional Néel state. As is the case with the CDVBS to Néel* transition discussed above, a continuous P-VBS to Néel QCP is forbidden within LGW theory (by similar arguments, which we refrain from giving explicitly for brevity). In this particular case, however, an alert reader may note that the two phases appear to be very similar to those recently argued to be connected by a continuous but non-LGW continuous phase transition, deemed a Deconfined Quantum Critical Point (DQCP) 34

The analogy, however, is not complete. Significantly, the checkerboard lattice differs in detail from the square lattice discussed in Ref. 34 in its point symmetry group. In particular, a $\pi / 2$ rotation about a site of the lattice, a symmetry of the square lattice, is not a symmetry operation of the checkerboard lattice. We believe this symmetry distinction is sufficient to destabilize the putative DQCP. It is beyond the scope of this paper to fully recapitulate the arguments of Ref. 34, which would be necessary to explain this conclusion in a stand-alone fashion. Instead, we will sketch these arguments, assuming the reader will refer to Ref. 34 for further details.

The crucial, indeed defining, property of the DQCP is an emergent topological conservation law, exactly maintained at the critical fixed point. Specifically, "skyrmion number" is conserved by the fixed point theory. This is not true microscopically at the lattice level, but is an emergent feature of critical theory, as argued in Ref. 34. A crucial step in that argument is the remarkable identification (due to Haldane ${ }^{35}$ ) of the skyrmion creation operator with the columnar/plaquette VBS order parameter. These can be defined through a complex scalar field $\Psi$ (see Ref. 34 ). We emphasize that although $\Psi$ has 
the symmetries of the rather "conventional" VBS order parameter, it is not to be viewed as a nearly-free field in the LGW sense, but rather some "composite" operator in the critical field theory. Under the $\pi / 2$ site rotation above, one finds $\Psi \rightarrow i \Psi$, and consequently, for the square lattice, the skyrmion creation operator can appear only in the fourth order in the continuum field theory action for the square lattice antiferromagnet, i.e., as a perturbation of the form $S^{\prime}=\int d \tau d^{2} x \lambda_{4} \operatorname{Re} \Psi^{4}$. A variety of arguments indicate then, because of the large (fourth) power of $\Psi$ which appears, $\lambda_{4}$ is irrelevant at the DQCP. On the checkerboard lattice, lacking this $\pi / 2$ site rotation, the remaining symmetries of the system are insufficient to rule out the much more relevant "quadratic" term $S^{\prime}=\int d \tau d^{2} x \lambda_{2} \operatorname{Re} \Psi^{2}$. The presence of the non-zero $\lambda_{2}$ term is, incidentally, also tied to the only two-fold degeneracy of the P-VBS state, compared to the four-fold degeneracy of the columnar and plaquette VBS states on the square lattice.

A necessary and sufficient condition for the stability of this DQCP on the checkerboard lattice is thus the irrelevance of $\lambda_{2}$. Ultimately, this can be decided only by detailed numerical calculations of the scaling dimension of the $\Psi^{2}$ operator. Unpublished numerical results ${ }^{36}$ for the easy-plane deformation of the theory (which is more numerically tractable) suggest that it is in fact relevant. If this conclusion is, as we suspect, true for the $\mathrm{SU}(2)$ symmetric model, the DQCP is not stable on the checkerboard lattice. Thus we are led to conclude that there is no viable candidate theory for a continuous Néel to PVBS quantum critical point in this model, and that such a transition is quite unlikely.

The simple harmonic analysis of the previous section predicts another "soft mode" transition in the $O(3)$ universality class out of the quadrumerized VBS state to one with Néel order at $J / J_{\times}<1$. The resulting magnetically ordered phase is, by its very construction, a coexistence region with both P-VBS and Néel order, that is, with less symmetry than either phase. This is built into the quadrumer boson expansion because all excitations are constructed about a background that explicitly has the reduced symmetry of the P-VBS state, and there is no mechanism to restore the full point group symmetries of the checkerboard lattice. Thus we believe the alternative possibility of a direct first-order (since the DQCP theory is unstable) transition from the P-VBS to the true Néel state should not be ruled out as a possibility. The possible existence of a coexistence region between VBS and Néel orders in various models is still a subject of some contention. It has been discussed in great detail in Ref. 37. An exact diagonalization study of the quantum checkerboard antiferromagnet, 11 has concluded that, if present at all, the co-existence phase is very narrow. Clearly more detailed studies of this interesting question are needed. At present, we can only reiterate that a single continuous transition is highly unlikely in view of the arguments presented above.

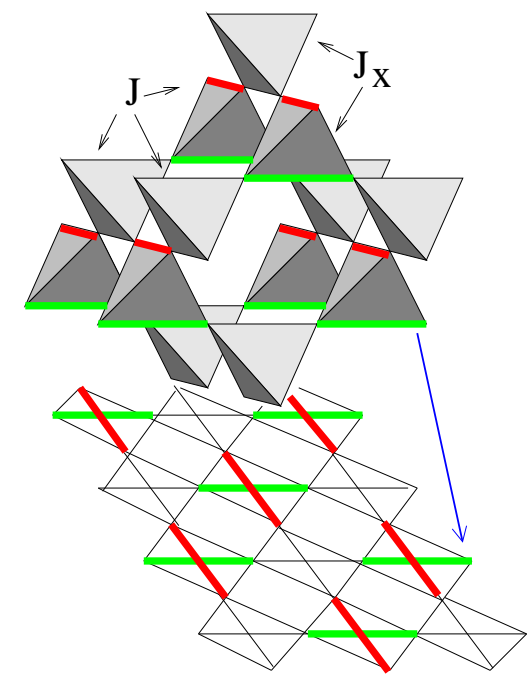

FIG. 7: (Color online) Three-dimensional dimerization pattern of a generalized "quasi-one-dimensional" pyrochlore antiferromagnet. Also shown, by a long blue arrow, is its "twodimensional" projection, which coincides with the crosseddimer order of Fig. 2

\section{BACK TO THREE DIMENSIONS}

Consider now the 3D pyrochlore. Although all bonds of a tetrahedron are equivalent by symmetry, it makes sense to ask, in analogy with the 2D lattice that we analyzed in this paper, what would happen if some bonds were stronger than others. The particular generalization motivated by the present study of the $2 \mathrm{D}$ projected model (which can be thought of as a "shadow" of the $3 \mathrm{D}$ pyrochlore on a $2 \mathrm{D}$ plane), involves a modified model in which two opposite bonds of tetrahedron are strong $(J)$ whereas four remaining bonds, connecting the strong ones, are weak $\left(J_{\times}\right)$. Then, in the limit $J_{\times} / J \ll 1$, one is back to the problem of strong spin chains coupled by weak and frustrated inter-chain $J_{\times}$. Now, however, chains are arranged in layers: chains are parallel to each other (oriented along either $x$ or $y$ direction) in each layer, but are orthogonal to those in the layers right above and below. That is, chains form a stack of the type $x-y-x-y \ldots$ along the vertical $(z)$ direction. Chains in one layer do not interact with each other, $J_{\times}$couples orthogonal chains from neighboring layers. This is just a $3 \mathrm{D}$ generalization of $2 \mathrm{D}$ situation analyzed in this paper. It does not introduce any new features, and hence the answer for the ground state is straightforward - it is spontaneously dimerized into the pattern shown in Fig. [7

Such a generalization is not unrealistic. It appears that $S=1 / 2$ pyrochlore material $\mathrm{GeCu}_{2} \mathrm{O}_{4}$ has exactly such a quasi-one-dimensional structure, 38 thanks to a strong Jahn-Teller elongation of $\mathrm{CuO}_{6}$ octahedra along the crystal $c$ direction ${ }^{38}$ From the high-temperature tail of the uniform spin susceptibility one can estimate the ratio of (frustrated) inter-chain exchange $J_{\times}$to the intra-chain one $J$ as $J_{\times} / J \approx 0.16 \stackrel{38}{=}$ At lower temperatures the uni- 
form spin susceptibility follows that of the spin chain down to $T_{c}=33 \mathrm{~K}$, where a small discontinuity is observed. The specific heat shows a sharp peak at the same temperature, suggesting a first-order transition to the magnetically ordered state of yet unknown structure (as far as we know, Ref. 38 is the only experimental study of this interesting material at the moment). The theory presented in this paper predicts that for a sufficiently small $J_{\times} / J$ ratio, the ordered state will be replaced by the quantum-disordered valence-bond solid shown in Fig. 7

Another very interesting realization of "onedimensionality" in the 3D setting is provided by the $S=1$ pyrochlore material $\mathrm{ZnV}_{2} \mathrm{O}_{4} \stackrel{39}{\text { There spin }}$ chains are formed below the structural orbital-ordering transition at $T_{c 1}=50 \mathrm{~K}$, as observed in the recent neutron scattering experiment 40 This is followed by the second, magnetic, transition at $T_{c 2}=40 \mathrm{~K}$. The resulting collinear magnetic order can be described as follows: $S=1$ spins order antiferromagnetically along the strong (chain) directions in such a way that spins along weaker $\left(J_{\times}\right)$bonds form a "4-spin" pattern, 41 "up-up-down-down-up-up-..." This 3D structure is, in fact, rather similar to the 2D one, Fig. 4 found in Sec. VB observe that spins on the inter-chain bonds in that Figure follow the same "4-spin" pattern of two up and two down spins. This is not a coincidence - in both cases the classical order can be understood in terms of the well-known "order-from-disorder" phenomenon, 42 induced by either quantum ${ }^{3}$ or thermal ${ }^{41}$ fluctuations. This analogy suggests that magnetically ordered state of $\mathrm{GeCu}_{2} \mathrm{O}_{4}$, observed in Ref. 38, should be similar to that in the low-temperature phase of $\mathrm{ZnV}_{2} \mathrm{O}_{4}$ - clearly more experimental and theoretical studies of this question are required. The analogy does not apply in the $J_{\times} / J \rightarrow 0$ limit where decoupled $S=1$ chains, although in the quantum-disordered phase with a finite spin gap $\Delta \sim 0.4 J, 43$ do not break translational symmetry. This is in contrast with $S=1 / 2$ chains studied in this paper; the decoupled limit is characterized by the crossed-dimer order, Fig. 7 which does break translational symmetry. Properties of the 3D phase transitions between quantum-disordered and ordered phases constitute another interesting theoretical problem which we leave for future studies.

\section{CONCLUSIONS}

The main result of this work consists in the prediction of the novel VBS phase, the crossed-dimer phase, illustrated in Fig. 2 and Fig. 7 This new VBS phase arises in the 1D limit of the model as a result of the frustration-fostered competition between classical (represented by the staggered magnetization $\vec{N}$ ) and quantum (represented by the staggered dimerization $\epsilon$ ) ordering tendencies. Our analysis is based on the careful perturbative implementation of the well-known $S U(2)$ symmetry of the $g$ matrix field of the WZW model, which provides rigorous field-theoretical description of the low-energy sector of the $S=1 / 2$ isotropic Heisenberg chain. This symmetry is made explicit by the OPE Eqs. (25)- 28), which show transformation properties of the low-energy "quantum triad" $\left\{\vec{J}_{R / L}, \vec{N}, \epsilon\right\}$. As shown in Sec. III consistent implementation of these OPEs requires a careful treatment of the often-neglected gradient terms ("nonprimary fields" in the conformal field theory nomenclature, such as $\left.\partial_{x} \vec{N}\right)$ which link together quantum fluctuations of the conserved spin current with those of the staggered magnetization and dimerization fields. Once this is done, straightforward inter-chain perturbation theory leads to the frustration-generated interaction of dimerizations on the crossing chains, Eq. (40).

Our finding of the CD-VBS phase in the $J_{\times} \ll J$ limit of the checkerboard antiferromagnet eliminates a previously proposed $^{8}$ sliding Luttinger liquid phase as the candidate for the ground state. Like many others, that work ${ }^{8}$ overlooked the crucial role of the gradient terms in the analysis of the frustrated inter-chain interaction between critical $S=1 / 2$ Heisenberg chains.

It is also worth pointing out here that our calculation clarifies previous somewhat inconclusive "sightings" of the decoupled-chains phase ${ }^{4.6}$ that arise in a widely-used large- $N$ approach ${ }^{44}$ to frustrated spin models. By its very construction, that technique fails to account, at the leading $N=\infty$ level, for the fluctuation-generated residual dimer-dimer interaction in the anisotropic 1D limit (although one does expect finite $1 / N$ corrections to the inter-dimer interaction to appear once the fluctuations of the compact gauge field are accounted for $\stackrel{4}{=})$

We have also presented a global phase diagram of the CCM (Sec. V). Although phenomenological in nature, our analysis stresses the importance of lattice symmetries in delineating the order of possible direct transitions between various quantum (CD- and P-VBS) and classical (ordered Néel and Néel*) phases of the CCM found in this and previous studies. We find that most of such transitions are required to be of the first-order type, or proceed via an intermediate co-existence phases, as illustrated in Figs. 5]and 6] This claim concerns even the relatively well studied P-VBS to Néel transition ${ }^{11,12,14}$ and clearly calls for more numerical (as well as analytical) investigations of this interesting question.

Last but not least, we have also presented a simple but intriguing extension of the approach to the anisotropic three-dimensional pyrochlore antiferromagnet, which may be relevant to both $S=1 / 2$ and $S=1$ pyrochlore-based magnetic materials ${ }^{38,40}$ We hope that this interesting connection will inspire new experiments in this exciting area.

\section{Acknowledgments}

We would like to thank A. Abanov, P. Azaria, I. Affleck, W. Brenig, F.H.L. Essler, M.P.A. Fisher, T. Fukui, F.D.M. Haldane, P. Lecheminant, R. Moessner, 
O. Motrunich, A.A. Nersesyan, S. Sachdev, P. Sindzingre, O. Tchernyshyov, H. Tsunetsugu, A.M. Tsvelik, and A. Vishwanath for discussions on questions related to this investigation. We are grateful to $\mathrm{O}$. Tchernyshyov for the help with Fig. 7

We thank Aspen Center for Physics, Kavli Institute for Theoretical Physics at UC Santa Barbara and Yukawa Institute for Theoretical Physics at Kyoto University for their hospitality during various stages of this research. O.A.S. thanks Research Corporation (award CC5491) for the partial support of this research. The work of A.F. was in part supported by a Grant-in-Aid for Scientific Research (No. 16GS50219) from Ministry of Education, Culture, Sports, Science and Technology, Japan. L.B. was supported by the NSF under grant DMR-9985255 and by the Packard Foundation.

\section{APPENDIX A: CORRELATION FUNCTIONS}

\section{Fermionic formulation}

Formally, the WZW model in Eqs. (9) and (19) describes all the low-energy properties of the $S=1 / 2$ Heisenberg spin chains, and is the starting point for a perturbative analysis of interchain coupling terms. Unfortunately, as remarked in Sec. ШB this action and Hamiltonian, while compact and self-contained, are not directly useful for concrete calculations. Indeed, in twodimensional conformal field theory, analysis often proceeds without any action or Hamiltonian, solely on the basis of algebraic operator product relations that essentially specify the field content and correlation functions of the theory. We will ultimately proceed by using operator product relations along these lines. In the crossed chains model, however, due to the local nature of interactions between perpendicular chains, we need to pay particular attention to proper short distance regularization of the theory. It is therefore desirable to have a more concrete formulation of the theory.

To do so, we use the well-known phenomena of spincharge separation in one-dimensional spin-1/2 Dirac fermions. In particular, using bosonization, one may show that all degrees of freedom (all states and operators) of such Dirac fermions are identical to those of a free scalar "charge" boson and a spin sector described by the WZW $S U(2)_{1}$ theory of interest to us. Moreover, the free Dirac fermion Hamiltonian,

$$
H_{d}=i v \int d x\left(\Psi_{L, s}^{\dagger} \partial_{x} \Psi_{L, s}-\Psi_{R, s}^{\dagger} \partial_{x} \Psi_{R, s}\right),
$$

can be expressed as the sum of decoupled spin and charge Hamiltonians, $H_{d}=H_{W Z W}+H_{\rho}$, with

$$
H_{\rho}=\frac{v}{2} \int d x\left[\left(\partial_{x} \varphi_{\rho}\right)^{2}+\left(\partial_{x} \vartheta_{\rho}\right)^{2}\right],
$$

where $\varphi_{\rho}, \vartheta_{\rho}$ are conjugate "charge" boson fields $\left[\varphi_{\rho}(x), \vartheta_{\rho}\left(x^{\prime}\right)\right]=-i \operatorname{sign}\left(x-x^{\prime}\right) / 2$. The right-moving and left-moving fermion operators may, if desired, themselves be re-expressed in terms of the gapless spin and charge fluctuations, which is commonly and conveniently done with the help of abelian bosonization ${ }^{23}$ We do not, however, require these expressions here.

Because the spin degrees of freedom in $H_{W Z W}$ (described by the $S U(2)$-valued field $g$ ) are independent of the charge boson fields, we may safely replace $H_{W Z W}$ by $H_{d}$ at the price of enlarging the physical Hilbert space to include these charge degrees of freedom, without however affecting the spin physics in any way. In particular, regardless of the spin interactions we add to $H_{W Z W}$, the charge sector remains always in its ground state, the vacuum of Eq. A2 .

The fermionic formulation provides a convenient way to calculate while simultaneously regularizing the shortdistance properties of the theory. This is accomplished by representing the important operators in the spin sector in terms of the Dirac fermions. First, the spin currents may be fully and simply re-expressed in terms of the Dirac fermions:

$$
\vec{J}_{R}=\Psi_{R, s}^{\dagger} \frac{\vec{\sigma}_{s, s^{\prime}}}{2} \Psi_{R, s^{\prime}}, \quad \vec{J}_{L}=\Psi_{L, s}^{\dagger} \frac{\vec{\sigma}_{s, s^{\prime}}}{2} \Psi_{L, s^{\prime}} .
$$

We can thereby construct the uniform magnetization, $\vec{J}=\vec{J}_{R}+\vec{J}_{L}$ in terms of fermions. Observe that $\vec{J}$ is invariant under chiral $U(1)$ charge symmetry

$$
\Psi_{R / L, s} \rightarrow e^{i \chi_{R / L}} \Psi_{R / L, s}
$$

with independent phases $\chi_{R} \neq \chi_{L}$. This invariance implies independence of $\vec{J}$ from the charge sector, Eq. A2, no matter what its precise form is.

The second important operator in the spin sector is the staggered magnetization. Unlike the uniform magnetization, however, it does not have a simple expression in terms of the fermions. Instead, one may define a fermionic "staggered" (corresponding to the $2 k_{F}$ component for physical electrons in the Tomonaga-Luttinger model) spin density:

$$
\vec{N}_{F}=\Psi_{R, s}^{\dagger} \frac{\vec{\sigma}_{s, s^{\prime}}}{2} \Psi_{L, s^{\prime}}+\Psi_{L, s}^{\dagger} \frac{\vec{\sigma}_{s, s^{\prime}}}{2} \Psi_{R, s^{\prime}}
$$

Using standard bosonization relations, one finds

$$
\vec{N}_{F}=\vec{N} \cos \sqrt{2 \pi} \varphi_{\rho} .
$$

Hence the fermionic staggered spin density, Eq. A5, reproduces the desired staggered magnetization, $\vec{N}$, but multiplied by a factor involving the charge boson $\varphi_{\rho}$.

Observe that $\vec{N}_{F}$ is not invariant under the chiral charge symmetry Eq. (A4) and this implies that it does couple to the charge sector of Eq. A1], as is explicitly stated in Eq. A6 above. (It is invariant under the diagonal subgroup of Eq. A4 with $\chi_{R}=\chi_{L}$ which only reflects that it conserves the total charge of the system, but not the charges of right- and left- moving chiral sectors independently.) 
Similarly, we may define a fermionic staggered dimerization operator,

$$
\epsilon_{F}=\frac{i}{2}\left(\Psi_{R, s}^{\dagger} \Psi_{L, s}-\Psi_{L, s}^{\dagger} \Psi_{R, s}\right) .
$$

Bosonization gives

$$
\epsilon_{F}=\epsilon \cos \sqrt{2 \pi} \varphi_{\rho}
$$

Like for the staggered magnetization, $\epsilon_{F}$ defined from the Dirac fermions reproduces the staggered dimerization operator $\epsilon$ in the spin sector, but with an undesired multiplicative charge factor. Here we observe again that this multiplicative charge factor follows from the fact that $\epsilon_{F}$ does not respect Eq. (A4), similarly to $\vec{N}_{F}$ above.

We will see below that, for our purposes this second factor in Eqs. A6 and A8 is innocuous, so that we may use $\vec{N}_{F}, \epsilon_{F}$ in place of the true $\vec{N}, \epsilon$ fields. We stress this is not generally true, and such a replacement is possible only under special circumstances we outline below. For example, because $\vec{N}_{F}, \epsilon_{F}$ contain charge fluctuations, their correlation functions are not the same as those of the $\vec{N}, \epsilon$ operators, and hence the latter cannot be evaluated using free fermion apparatus. However, the fermionic operators have the same operator products with the spin current field $\vec{J}$ as the corresponding spin operators. This is because the spin current, as discussed above, does not depend on charge sector, and as a result the "charge pieces" of $\vec{N}_{F}$ and $\epsilon_{F}$ stay "inert" during OPE calculations as outlined in Appendix B As it turns out, such operator products are all we need in order to solve the problem. Thus, we can evaluate these crucial operator products using those of the free fermions. Furthermore, we can regularize the problem by adopting a short-distance cutoff for the Dirac fermions. This is particularly convenient, as a simple ultraviolet regularization is available in the Dirac formulation which keeps $\mathrm{SU}(2)$ spin rotation symmetry manifest at all stages.

\section{Fermion Green's function.}

To proceed with our program we need then the Green's function of free fermions $\Psi_{R / L}$. As the inter-chain interaction, Eq. 223), is the sum of local terms over crossings of the lattice, one needs to be careful about the highenergy regularization of the continuum theory. We have found that the most natural and physically appealing regularization is provided by the "soft-momentum cutoff" scheme ${ }^{45.46}$ In that scheme cutoff $a$ is introduced via $e^{-a|q|}$ factor in the mode expansion of collective bosonic fields. In going from the momentum to the coordinate space, cutoff dependence on $a$ transforms into that on $\alpha \equiv a / v$ in the temporal $(\tau)$ direction. Adopting this scheme, one finds that the zero temperature $(T=0)$ Green's function for the right-moving fermions on the same chain (chain index is omitted for brevity) is given by

$$
\begin{aligned}
F_{R}(x, \tau) & =-\left\langle\hat{T} \Psi_{R, s}(x, \tau) \Psi_{R, s}^{+}(0,0)\right\rangle \\
& =-\frac{1}{2 \pi v\left(\tau-i x / v+\alpha \sigma_{\tau}\right)}
\end{aligned}
$$

where $\alpha=a / v$ is the ultraviolet cutoff in temporal direction and $\sigma_{\tau}=\operatorname{sign}(\tau)=\Theta(\tau)-\Theta(-\tau)$ is the signfunction. For the left-moving ones

$$
\begin{aligned}
F_{L}(x, \tau) & =F_{R}^{*}(x, \tau)=F_{R}(-x, \tau)=-F_{R}(x,-\tau) \\
& =-\frac{1}{2 \pi v\left(\tau+i x / v+\alpha \sigma_{\tau}\right)} .
\end{aligned}
$$

It is often convenient to introduce complex coordinates

$$
z=\tau+i x / v, \quad \bar{z}=\tau-i x / v
$$

in terms of which

$$
F_{R}(\bar{z})=-\frac{1}{2 \pi v \bar{z}}, \quad F_{L}(z)=-\frac{1}{2 \pi v z} .
$$

It is important to keep in mind, however, that expressions of Eqs. A9 and (A10) are "superior" to Eq. A12 in that they contain an explicit cutoff prescription.

\section{APPENDIX B: DERIVATION OF OPE EQ. (27)}

We need Eqs. A3 and A5 and the single-particle Green's function, Eqs. A9 and A10. Consider the product of $J_{R}^{a}\left(x_{1}, \tau_{1}\right)$ and $N_{F}^{b}\left(x_{2}, \tau_{2}\right)$ at nearby points (such that $\left|z_{1}-z_{2}\right| \sim \alpha$ with $z_{i}=\tau_{i}+i x_{i} / v$ ). To shorten notations, we denote $\left(x_{1}, \tau_{1}\right)=\mathbf{1}$, etc. Now use Wick's theorem for free fermions, as appropriate for Eq. A1, as well as the identity $\sigma^{a} \sigma^{b}=\delta^{a b}+i \epsilon^{a b c} \sigma^{c}$, to reduce the product of four $\Psi$ 's to that of two of them and $F_{R}$

$$
\begin{aligned}
& J_{R}^{a}(\mathbf{1}) N_{F}^{b}(\mathbf{2})=-\frac{1}{4} F_{R}(\mathbf{1}-\mathbf{2}) \\
& \times\left(i \epsilon^{a b c} \sigma_{s, s^{\prime}}^{c}\left[\Psi_{R, s}^{+}(\mathbf{1}) \Psi_{L, s^{\prime}}(\mathbf{2})+\Psi_{L, s}^{+}(\mathbf{1}) \Psi_{R, s^{\prime}}(\mathbf{2})\right]\right. \\
& \left.\quad+\delta^{a b}\left[\Psi_{R, s}^{+}(\mathbf{1}) \Psi_{L, s}(\mathbf{2})-\Psi_{L, s}^{+}(\mathbf{1}) \Psi_{R, s}(\mathbf{2})\right]\right),(\mathrm{B} 1)
\end{aligned}
$$

where summation over repeated spin indices $s$ and $s^{\prime}$ is assumed. Fusing $\left(x_{1}, \tau_{1}\right)$ and $\left(x_{2}, \tau_{2}\right)$ points, one finds that the coefficient of $\epsilon^{a b c}$ is just $2 N_{F}^{c}(\mathbf{2})$, whereas that of $\delta^{a b}$ is $-2 i \epsilon_{F}(\mathbf{2})$, the staggered dimerization operator; see Eq. A7]. Hence

$$
J_{R}^{a}(\mathbf{1}) N_{F}^{b}(\mathbf{2})=\frac{i \epsilon^{a b c} N_{F}^{c}(\mathbf{2})-i \delta^{a b} \epsilon_{F}(\mathbf{2})}{4 \pi v\left(\bar{z}_{1}-\bar{z}_{2}+\alpha \sigma_{\tau_{1}-\tau_{2}}\right)} .
$$

The second OPE, between $\vec{J}_{L}$ and $\vec{N}_{F}$, is obtained by replacing $F_{R} \rightarrow F_{L}$ and changing the sign of the last term in Eq. (B2) as is readily verified by the explicit calculation. Thus

$$
J_{L}^{a}(\mathbf{1}) N_{F}^{b}(\mathbf{2})=\frac{i \epsilon^{a b c} N_{F}^{c}(\mathbf{2})+i \delta^{a b} \epsilon_{F}(\mathbf{2})}{4 \pi v\left(z_{1}-z_{2}+\alpha \sigma_{\tau_{1}-\tau_{2}}\right)} .
$$


Observe now that the multiplicative charge factor $\cos \sqrt{2 \pi} \varphi_{\rho}$ from Eqs. A6 and (A8) appears on both sides of Eqs. (B2) and (B3) above. Dividing by it, we obtain the OPE quoted in Eq. (27)

$$
\begin{aligned}
& J_{R}^{a}(\bar{z}) N^{b}(w, \bar{w})=\frac{i \epsilon^{a b c} N^{c}(w, \bar{w})-i \delta^{a b} \epsilon(w, \bar{w})}{4 \pi v(\bar{z}-\bar{w})}, \\
& J_{L}^{a}(z) N^{b}(w, \bar{w})=\frac{i \epsilon^{a b c} N^{c}(w, \bar{w})+i \delta^{a b} \epsilon(w, \bar{w})}{4 \pi v(z-w)} .
\end{aligned}
$$

It is instructive to repeat the same calculation using abelian bosonization. Consider, for example, $J_{R}^{z}\left(x_{1}, \tau_{1}\right) N^{x}\left(x_{2}, \tau_{2}\right)$. Abelian bosonization tells us that

$$
\begin{aligned}
J_{R}^{z}(\mathbf{1}) & =\frac{1}{\sqrt{2 \pi}} \partial_{x_{1}} \Phi_{R, \sigma}(\mathbf{1}) \\
N^{x}(\mathbf{2}) & =\frac{\lambda}{4 \pi a}\left(e^{-i \sqrt{2 \pi}\left[\Phi_{R, \sigma}(\mathbf{1})-\Phi_{L, \sigma}(\mathbf{1})\right]}+\text { H.c. }\right),
\end{aligned}
$$

where $\Phi_{R / L, \sigma}$ are the spin components of chiral right/left bosons, and the scale factor $\lambda=\left\langle\cos \sqrt{2 \pi} \varphi_{\rho}\right\rangle_{\rho}$ is the expectation value of the charge field. The brackets $\langle\ldots\rangle_{\rho}$ denote average with respect to the charge Hamiltonian $H_{\rho}^{\prime}$ which includes now four-fermion umklapp term responsible for the opening of the charge gap (as a result of which $\lambda \neq 0$ ), see Appendix A of Ref. 27 for more details.
Thus charge fluctuations are explicitly separated from spin ones in the manipulations to follow. To fuse Eq. (B5) and Eq. (B6), we need

$$
\Phi_{R, \sigma}(\mathbf{1}) e^{i \beta \Phi_{R, \sigma}(\mathbf{2})}=i \beta\left\langle\hat{T} \Phi_{R, \sigma}(\mathbf{1}) \Phi_{R, \sigma}(\mathbf{2})\right\rangle e^{i \beta \Phi_{R, \sigma}(\mathbf{2})},
$$

which is obtained by expanding exponential on the lefthand side of Eq. (B7), pairing $\Phi_{R, \sigma}(\mathbf{1})$ with $\Phi_{R, \sigma}(\mathbf{2})$ in all possible ways (Wick's theorem for free chiral spin bosons), and collecting the rest of the series back into exponential. Using Eq. (B7) and the fact that right and left bosons are independent from each other, we find

$$
J_{R}^{z}(\mathbf{1}) N^{x}(\mathbf{2})=\partial_{x_{1}}\left\langle\hat{T} \Phi_{R, \sigma}(\mathbf{1}) \Phi_{R, \sigma}(\mathbf{2})\right\rangle N^{y}(\mathbf{2}) .
$$

Finally,

$$
\left\langle\hat{T} \Phi_{R, \sigma}(\mathbf{1}) \Phi_{R, \sigma}(\mathbf{2})\right\rangle=-\frac{1}{4 \pi} \ln \left[\alpha+\sigma_{\tau_{1}-\tau_{2}}\left(\bar{z}_{1}-\bar{z}_{2}\right)\right]
$$

for free chiral bosons. Hence

$$
J_{R}^{z}(\mathbf{1}) N^{x}(\mathbf{2})=\frac{i N^{y}(\mathbf{2})}{4 \pi v\left(\bar{z}_{1}-\bar{z}_{2}+\alpha \sigma_{\tau_{1}-\tau_{2}}\right)},
$$

which is just one component of Eq. (B2). To get $\delta^{a b}$ term of Eq. (B2) one has to consider explicitly OPE of, say, $J^{z}$ and $N^{z}$ fields.
1 R.R.P. Singh, O.A. Starykh, and P.J. Freitas, J. Appl. Phys. 83, 7387 (1998).

2 B. Canals, Phys. Rev. B 65, 184408 (2002).

3 O. Tchernyshyov, O.A. Starykh, R. Moessner, and A.G. Abanov, Phys. Rev. B 68, 144422 (2003).

4 C.H. Chung, J.B. Marston, and S. Sachdev, Phys. Rev. B 64, 134407 (2001).

${ }^{5}$ R. Moessner, O. Tchernyshyov, and S.L. Sondhi, J. Stat. Phys. 116, 755 (2004); cond-mat/0106286

6 J.S. Bernier, C.H. Chung, Y.B. Kim, and S. Sachdev, Phys. Rev. B 69, 214427 (2004).

7 M. Hermele, M.P.A. Fisher, and L. Balents, Phys. Rev. B 69, 064404 (2004).

8 O.A. Starykh, R.R.P. Singh, and G.C. Levine, Phys. Rev. Lett. 88, 167203 (2002).

9 S.E. Palmer and J.T. Chalker, Phys. Rev. B 64, 094412 (2001).

10 J.B. Fouet, M. Mambrini, P. Sindzingre, and C. Lhuillier, Phys. Rev. B 67, 054411 (2003).

11 P. Sindzingre, J.B. Fouet, and C. Lhuillier, Phys. Rev. B 66, 174421 (2002).

12 W. Brenig and A. Honecker, Phys. Rev. B 65, 140407 (2002).

13 E. Berg, E. Altman, and A. Auerbach, Phys. Rev. Lett. 90, 147204 (2003).

14 W. Brenig and M. Grzeschik, Phys. Rev. B 69, 064420 (2004).

15 O.A. Starykh and L. Balents, Phys. Rev. Lett. 93, 127202 (2004).
16 I. Affleck and B.I. Halperin, J. Phys. A: Math. Gen. 29, 2627 (1996).

17 H. Schulz, Phys. Rev. Lett. 77, 2790 (1996).

18 D. Allen, F.H.L. Essler, and A.A. Nersesyan, Phys. Rev. B 61, 8871 (2000).

19 E.H. Kim, G. Fáth, J. Sólyom, and D.J. Scalapino, Phys. Rev. B 62, 14965 (2000).

20 A.A. Nersesyan and A.M. Tsvelik, Phys. Rev. B 67, 024422 (2003).

21 I. Affleck and F.D.M. Haldane, Phys. Rev. B 36, 5291 (1987).

22 C. Itzykson and J.-M. Drouffe, Statistical Field Theory, vol. 2, Appendix 9C (Cambridge University Press, Cambridge, UK, 1989).

23 A.O. Gogolin, A.A. Nersesyan, and A.M. Tsvelik, Bosonization and Strongly Correlated Systems (Cambridge University Press, Cambridge, UK, 1998).

24 S. Eggert and I. Affleck, Phys. Rev. B 46, 10866 (1992).

25 H.-H. Lin, L. Balents, and M.P.A. Fisher, Phys. Rev. B 56, 6569 (1997).

26 I. Affleck, private communication.

27 D.G. Shelton, A.A. Nersesyan and A.M. Tsvelik, Phys. Rev. B 53, 8521 (1996).

28 S. Lukyanov and A. Zamolodchikov, Nucl. Phys. B 493, 571 (1997).

29 O.A. Starykh, M.E. Zhitomirsky, D.I. Khomskii, R.R.P. Singh, and K. Ueda, Phys. Rev. Lett. 77, 2558 (1996).

30 M.E. Zhitomirsky and K. Ueda, Phys. Rev. B 54, 9047 (1996). 
31 A. Läuchli, S. Wessel, and M. Sigrist, Phys. Rev. B 66, 014401 (2002).

32 We thank W. Brenig for the discussions on this point.

33 L.D. Landau and E.M. Lifshits, Statistical Physics, Part 1, 3rd Edition (Butterworth-Heinemann, New York, 2003).

${ }^{34}$ T. Senthil, L. Balents, S. Sachdev, A. Vishwanath, and M.P.A. Fisher, Phys. Rev. B 70, 144407 (2004).

35 F.D.M. Haldane, Phys. Rev. Lett. 61, 1029 (1988).

36 O.I. Motrunich, private communication.

37 S. Sachdev and K. Park, Ann. Phys. (N.Y.) 298, 58 (2002).

38 T. Yamada, Z. Hiroi, M. Takano, M. Nohara, and H. Takagi, J. Phys. Soc. Jpn. 69, 1477 (2000).

39 Y. Ueda, N. Fujiwara, and H. Yasuoka, J. Phys. Soc. Jpn. 66, 778 (1997).

40 S.-H. Lee, D. Louca, H. Ueda, S. Park, T.J. Sato, M. Isobe,
Y. Ueda, S. Rosenkranz, P. Zschack, J. Iniguez, Y. Qiu, and R. Osborn, Phys. Rev. Lett. 93, 156407 (2004).

41 Y. Motome and H. Tsunetsugu, Phys. Rev. B 70, 184427 (2004); H. Tsunetsugu and Y. Motome, Phys. Rev. B 68, 060405 (2003).

42 E.F. Shender, Sov. Phys. JETP 56, 178 (1982); C.L. Henley, Phys. Rev. Lett. 62, 2056 (1989).

43 F.D.M. Haldane, Phys. Lett. 93A, 464 (1983); Phys. Rev. Lett. 50, 1153 (1983).

44 N. Read and S. Sachdev, Phys. Rev. B 42, 4568 (1990).

45 F.D.M. Haldane, J. Phys. C 14, 2585 (1980).

46 J. von Delft and H. Schoeller, Ann. Phys. (Leipzig) 7, 225 (1998). 